\title{
Hybrid steepest-descent viscosity methods for triple hierarchical variational inequalities with constraints of mixed equilibria and bilevel variational inequalities
}

\author{
Lu-Chuan Ceng ${ }^{\mathrm{a}}$, Yeong-Cheng Liou ${ }^{\mathrm{b}}$, Ching-Feng Wen ${ }^{\mathrm{c}, *}$, Abdul Latif ${ }^{\mathrm{d}}$ \\ ${ }^{a}$ Department of Mathematics, Shanghai Normal University; and Scientific Computing Key Laboratory of Shanghai Universities, \\ Shanghai 200234, China. \\ ${ }^{b}$ Department of Healthcare Administration and Medical Informatics; and Research Center for Nonlinear Analysis and Optimization, \\ Kaohsiung Medical University, Kaohsiung 80702, Taiwan. \\ ${ }^{c}$ Center for Fundamental Science; and Research Center for Nonlinear Analysis and Optimization, Kaohsiung Medical University, \\ Kaohsiung 80702, Taiwan; Department of Medical Research, Kaohsiung Medical University Hospital, Kaohsiung 80702, Taiwan. \\ ${ }^{d}$ Department of Mathematics, King Abdulaziz University, P. O. Box 80203, Jeddah 21589, Saudi Arabia.
}

Communicated by Y. H. Yao

\begin{abstract}
In this paper, we introduce and analyze a hybrid steepest-descent viscosity algorithm for solving the triple hierarchical variational inequality problem with constraints of two problems: one generalized mixed equilibrium problem and another bilevel variational inequality problem in a real Hilbert space. Under mild conditions, the strong convergence of the iteration sequences generated by the algorithm is established. Our results improve and extend the corresponding results in the earlier and recent literature. (C)2017 All rights reserved.
\end{abstract}

Keywords: Hybrid steepest-descent viscosity method, triple hierarchical variational inequality, generalized mixed equilibrium problem.

2010 MSC: 49J30, 47H09, 47J20, 49M05.

\section{Introduction}

Let $\mathrm{H}$ be a real Hilbert space with inner product $\langle\cdot, \cdot\rangle$ and norm $\|\cdot\|, \mathrm{C}$ be a nonempty closed convex subset of $H$, and $P_{C}$ be the metric projection of $H$ onto $C$. If $\left\{x_{n}\right\}$ is a sequence in $H$, then we denote by $x_{n} \rightarrow x$ (respectively, $x_{n} \rightarrow x$ ) the strong (respectively, weak) convergence of the sequence $\left\{x_{n}\right\}$ to $x$. Let $\mathrm{S}: \mathrm{C} \rightarrow \mathrm{H}$ be a nonlinear mapping on $\mathrm{C}$. We denote by Fix $(\mathrm{S})$ the set of fixed points of $\mathrm{S}$ and by $\mathbf{R}$ the set of all real numbers. A mapping $\mathrm{S}: \mathrm{C} \rightarrow \mathrm{H}$ is called L-Lipschitz continuous if there exists a constant $\mathrm{L} \geqslant 0$ such that

$$
\|S x-S y\| \leqslant L\|x-y\|, \quad \forall x, y \in C
$$

\footnotetext{
*Corresponding author

Email addresses: zenglc@hotmail.com (Lu-Chuan Ceng), simplex_liou@hotmail.com (Yeong-Cheng Liou), cf wen@kmu.edu.tw (Ching-Feng Wen), alatif@kau.edu.sa (Abdul Latif)

doi:10.22436/jnsa.010.03.23
} 
In particular, if $L=1$, then $S$ is called a nonexpansive mapping; if $L \in[0,1)$, then $S$ is called a contraction.

Let $\mathcal{A}: \mathrm{C} \rightarrow \mathrm{H}$ be a nonlinear mapping. The classical variational inequality problem (VIP) $([10,15])$ is to find $x \in \mathrm{C}$ such that

$$
\langle\mathcal{A} x, y-x\rangle \geqslant 0, \quad \forall y \in C .
$$

The solution set of VIP $(1.1)$ is denoted by $\operatorname{VI}(C, \mathcal{A})$.

It is well-known that, if $A$ is a strongly monotone and Lipschitz-continuous mapping on $C$, then VIP (1.1) has a unique solution. In 1976, Korpelevich [14] proposed the following extragradient method for solving the VIP (1.1) in Euclidean space $\mathbf{R}^{\mathrm{n}}$ :

$$
\left\{\begin{array}{l}
y_{k}=P_{C}\left(x_{k}-\tau A x_{k}\right), \\
x_{k+1}=P_{C}\left(x_{k}-\tau A y_{k}\right), \quad \forall k \geqslant 0,
\end{array}\right.
$$

with $\tau>0$ a given number. The literature on the VIP is vast and Korpelevich's extragradient method has received great attention given by many authors, who improved it in various ways; see e.g., [4, 5, 18, 26, $27,31]$ and references therein.

Let $\mathrm{A}: \mathrm{C} \rightarrow \mathrm{H}$ and $\mathrm{B}: \mathrm{H} \rightarrow \mathrm{H}$ be two mappings. Consider the following bilevel variational inequality problem (BVIP):

Problem 1.1. We find $x^{*} \in \mathrm{VI}(\mathrm{C}, \mathrm{B})$ such that

$$
\left\langle A x^{*}, x-x^{*}\right\rangle \geqslant 0, \quad \forall x \in \operatorname{VI}(C, B),
$$

where $\operatorname{VI}(C, B)$ denotes the set of solutions of the VIP: find $y^{*} \in C$ such that

$$
\left\langle B y^{*}, y-y^{*}\right\rangle \geqslant 0, \quad \forall y \in C .
$$

In particular, whenever $H=\mathbf{R}^{n}$, the BVIP was recently studied by Anh et al. [1]. Bilevel variational inequalities are special classes of quasivariational inequalities $([9,24])$ and of equilibrium with equilibrium constraints considered in [13]. However it covers some classes of mathematical programs with equilibrium constraints, bilevel minimization problems ([19]), variational inequalities $([28,30])$ and complementarity problems.

In what follows, suppose that $A$ and $B$ satisfy the following conditions:

(C1) $B$ is pseudomonotone on $\mathrm{H}$ and $A$ is $\beta$-strongly monotone on $C$;

(C2) $A$ is $L_{1}$-Lipschitz continuous on $C$;

(C3) $\mathrm{B}$ is $\mathrm{L}_{2}$-Lipschitz continuous on $\mathrm{H}$;

(C4) $\operatorname{VI}(C, B) \neq \emptyset$.

In 2012, Anh et al. [1] introduced the following extragradient iterative algorithm for solving the above bilevel variational inequality.

Algorithm 1.2 ([1, Algorithm 2.1]). Initialization: choose $u \in \mathbf{R}^{\mathfrak{n}}$ and $x_{0} \in C$.

Step 1. Compute $y_{k}:=P_{C}\left(x_{k}-\lambda_{k} B x_{k}\right)$ and $z_{k}:=P_{C}\left(x_{k}-\lambda_{k} B y_{k}\right)$.

Step 2. Inner loop $j=0,1, \ldots$ Compute

$$
\left\{\begin{array}{l}
x_{k, 0}:=z_{k}-\lambda A z_{k}, \\
y_{k, j}:=P_{C}\left(x_{k, j}-\delta_{j} B x_{k, j}\right), \\
x_{k, j+1}:=\alpha_{j} x_{k, 0}+\beta_{j} x_{k, j}+\gamma_{j} P_{C}\left(x_{k, j}-\delta_{j} B y_{k, j}\right) . \\
\text { If }\left\|x_{k, j+1}-P_{V I(C, B)} x_{k, 0}\right\| \leqslant \bar{\epsilon}_{k} \text { then set } h_{k}:=x_{k, j+1} \text { and go to Step } 3, \\
\text { Otherwise, increase } j \text { by } 1 \text { and repeat the inner loop Step } 2 .
\end{array}\right.
$$

Step 3. Set $x_{k+1}:=\alpha_{k} u+\beta_{k} x_{k}+\gamma_{k} h_{k}$. Then increase $k$ by 1 and go to Step 1 . 
Theorem 1.3. Suppose that the assumptions (C1)-(C4) hold. Then the two sequences $\left\{x_{k}\right\}$ and $\left\{z_{k}\right\}$ in Algorithm 1.2 converge to the same point $x^{*}$ which is a solution of the BVI.

Furthermore, let $\varphi: \mathrm{C} \rightarrow \mathbf{R}$ be a real-valued function, $\mathcal{A}: \mathrm{H} \rightarrow \mathrm{H}$ be a nonlinear mapping and $\Theta: C \times C \rightarrow \mathbf{R}$ be a bifunction. In 2008, Peng and Yao [18] introduced the following generalized mixed equilibrium problem (GMEP) of finding $x \in C$ such that

$$
\Theta(x, y)+\varphi(y)-\varphi(x)+\langle\mathcal{A} x, y-x\rangle \geqslant 0, \quad \forall y \in C .
$$

We denote the set of solutions of $\operatorname{GMEP}(1.2)$ by $\operatorname{GMEP}(\Theta, \varphi, \mathcal{A})$. The $\operatorname{GMEP}(1.2)$ is very general in the sense that it includes, as special cases, optimization problems, variational inequalities, minimax problems, Nash equilibrium problems in noncooperative games and others. The GMEP is further considered and studied; see e.g., [7]. In particular, if $\varphi=0$, then GMEP (1.2) reduces to the generalized equilibrium problem (GEP) ([22]) which is to find $x \in C$ such that

$$
\Theta(x, y)+\langle\mathcal{A} x, y-x\rangle \geqslant 0, \quad \forall y \in C .
$$

The set of solutions of GEP is denoted by $\operatorname{GEP}(\Theta, \mathcal{A})$.

If $\mathcal{A}=0$, then GMEP (1.2) reduces to the mixed equilibrium problem (MEP) ([8]), which is to find $x \in \mathrm{C}$ such that

$$
\Theta(x, y)+\varphi(y)-\varphi(x) \geqslant 0, \quad \forall y \in C .
$$

The set of solutions of MEP is denoted by $\operatorname{MEP}(\Theta, \varphi)$.

If $\varphi=0, \mathcal{A}=0$, then GMEP (1.2) reduces to the equilibrium problem (EP) $([2,21])$, which is to find $x \in \mathrm{C}$ such that

$$
\Theta(x, y) \geqslant 0, \quad \forall y \in C .
$$

The set of solutions of EP is denoted by $\operatorname{EP}(\Theta)$. It is worth to mention that the EP is a unified model of several problems, namely, variational inequality problems, optimization problems, saddle point problems, complementarity problems, fixed point problems, Nash equilibrium problems, etc.

It is assumed as in [18] that $\Theta: C \times C \rightarrow \mathbf{R}$ is a bifunction satisfying conditions (A1)-(A4) and $\varphi: \mathbf{C} \rightarrow \mathbf{R}$ is a lower semicontinuous and convex function with restrictions (B1) or (B2), where

(A1) $\Theta(x, x)=0$ for all $x \in C$;

(A2) $\Theta$ is monotone, i.e., $\Theta(x, y)+\Theta(y, x) \leqslant 0$ for any $x, y \in C$;

(A3) $\Theta$ is upper-hemicontinuous, i.e., for each $x, y, z \in C$,

$$
\limsup _{t \rightarrow 0^{+}} \Theta(t z+(1-t) x, y) \leqslant \Theta(x, y)
$$

(A4) $\Theta(x, \cdot)$ is convex and lower semicontinuous for each $x \in C$;

(B1) for each $x \in H$ and $r>0$, there exists a bounded subset $D_{x} \subset C$ and $y_{x} \in C$ such that for any $z \in C \backslash D_{x}$,

$$
\Theta\left(z, y_{x}\right)+\varphi\left(y_{x}\right)-\varphi(z)+\frac{1}{r}\left\langle y_{x}-z, z-x\right\rangle<0
$$

(B2) $\mathrm{C}$ is a bounded set.

Given a positive number $r>0$. Let $T_{r}^{(\Theta, \varphi)}: H \rightarrow C$ be the solution set of the auxiliary mixed equilibrium problem, that is, for each $x \in H$,

$$
\mathrm{T}_{r}^{(\Theta, \varphi)}(x):=\left\{y \in C: \Theta(y, z)+\varphi(z)-\varphi(y)+\frac{1}{r}\langle y-x, z-y\rangle \geqslant 0, \forall z \in C\right\} .
$$

Recall that, a mapping $T: C \rightarrow C$ is said to be semicompact if for any bounded sequence $\left\{x_{n}\right\}$ in $C$ such that $\left\|x_{n}-T x_{n}\right\| \rightarrow 0$ as $n \rightarrow \infty$, there exists a subsequence $\left\{x_{n_{i}}\right\} \subset\left\{x_{n}\right\}$ such that $x_{n_{i}} \rightarrow x^{*}$. A 
mapping $\mathrm{T}: \mathrm{C} \rightarrow \mathrm{C}$ is called a $\zeta$-strictly pseudocontractive mapping (or a $\zeta$-strict pseudocontraction) if there exists a constant $\zeta \in[0,1)$ such that

$$
\|\mathrm{T} x-\mathrm{T} y\|^{2} \leqslant\|x-y\|^{2}+\zeta\|(\mathrm{I}-\mathrm{T}) x-(\mathrm{I}-\mathrm{T}) \mathrm{y}\|^{2}, \quad \forall x, y \in \mathrm{C} .
$$

In 2009, Ceng et al. [2] proposed an iterative scheme for finding a common element of the set of solutions of the EP (1.3) and the set of fixed points of a strictly pseudocontractive mapping in a real Hilbert space $\mathrm{H}$. They established some weak and strong convergence theorems by combining the ideas of Marino and Xu's result [16] and Takahashi and Takahashi's result [21].

Recall the variational inequality for a monotone operator $A_{1}: H \rightarrow H$ over the fixed point set of a nonexpansive mapping $\mathrm{T}: \mathrm{H} \rightarrow \mathrm{H}$ :

$$
\text { find } \bar{x} \in \operatorname{VI}\left(\operatorname{Fix}(T), A_{1}\right):=\left\{\bar{x} \in \operatorname{Fix}(T):\left\langle A_{1} \bar{x}, y-\bar{x}\right\rangle \geqslant 0, \forall y \in \operatorname{Fix}(T)\right\},
$$

where $\operatorname{Fix}(T):=\{x \in H: T x=x\} \neq \emptyset$. In [12], liduka introduced the following three-stage variational inequality problem, that is, the following monotone variational inequality with variational inequality constraint over the fixed point set of a nonexpansive mapping.

Problem 1.4. Assume that

(i) $\mathrm{T}: \mathrm{H} \rightarrow \mathrm{H}$ is a nonexpansive mapping with $\operatorname{Fix}(\mathrm{T}) \neq \emptyset$;

(ii) $A_{1}: H \rightarrow H$ is $\alpha$-inverse strongly monotone;

(iii) $A_{2}: H \rightarrow H$ is $\beta$-strongly monotone and L-Lipschitz continuous;

(iv) $\operatorname{VI}\left(\operatorname{Fix}(T), A_{1}\right) \neq \emptyset$.

Then the objective is to

$$
\text { find } x^{*} \in \operatorname{VI}\left(\operatorname{VI}\left(\operatorname{Fix}(T), A_{1}\right), A_{2}\right):=\left\{x^{*} \in \operatorname{VI}\left(\operatorname{Fix}(T), A_{1}\right):\left\langle A_{2} x^{*}, v-x^{*}\right\rangle \geqslant 0, \forall v \in \operatorname{VI}\left(\operatorname{Fix}(T), A_{1}\right)\right\} .
$$

Since this problem has a triple structure in contrast with bilevel programming problems ([17]) or hierarchical constrained optimization problems or hierarchical fixed point problem, it is referred to as a triple-hierarchical constrained optimization problem (THCOP). More precisely, it is referred as a triple hierarchical variational inequality problem (THVIP); see Ceng et al. [6]. Very recently, some authors continued the study of Iiduka's THVIP (i.e., Problem 1.4) and its variant and extension; see e.g., [3, 6, 29].

Algorithm 1.5 ([12, Algorithm 4.1]). Let $\mathrm{T}: \mathrm{H} \rightarrow \mathrm{H}$ and $A_{i}: \mathrm{H} \rightarrow \mathrm{H}(\mathrm{i}=1,2)$ satisfy the assumptions (i)-(iv) in Problem 1.4. The following steps are presented for solving Problem 1.4.

Step 0. Take $\left\{\alpha_{\mathrm{k}}\right\}_{\mathrm{k}=0}^{\infty},\left\{\lambda_{\mathrm{k}}\right\}_{\mathrm{k}=0}^{\infty} \subset(0, \infty)$, and $\mu>0$, choose $x_{0} \in \mathrm{H}$ arbitrarily, and let $\mathrm{k}:=0$.

Step 1. Given $x_{k} \in H$, compute $x_{k+1} \in H$ as

$$
\left\{\begin{array}{l}
y_{k}:=T\left(x_{k}-\lambda_{k} A_{1} x_{k}\right), \\
x_{k+1}:=y_{k}-\mu \alpha_{k} A_{2} y_{k} .
\end{array}\right.
$$

Update $k:=k+1$ and go to Step 1 .

Theorem 1.6 ([12, Theorem 4.1]). Assume that $\left\{y_{k}\right\}_{k=0}^{\infty}$ in Algorithm 1.5 is bounded. If $\mu \in\left(0, \frac{2 \beta}{\mathrm{L}^{2}}\right)$ is used and if $\left\{\alpha_{k}\right\}_{k=0}^{\infty} \subset(0,1]$ and $\left\{\alpha_{k}\right\}_{k=0}^{\infty} \subset(0,2 \alpha]$ satisfying (i) $\lim _{k \rightarrow \infty} \alpha_{k}=0$, (ii) $\sum_{k=0}^{\infty} \alpha_{k}=\infty$, (iii) $\sum_{k=0}^{\infty} \mid \alpha_{k+1}-$ $\alpha_{k} \mid<\infty$, (iv) $\sum_{k=0}^{\infty}\left|\lambda_{k+1}-\lambda_{k}\right|<\infty$, and (v) $\lambda_{k} \leqslant \alpha_{k} \forall k \geqslant 0$, are used, then the sequence $\left\{x_{k}\right\}_{k=0}^{\infty}$ generated by Algorithm 1.5 satisfies the following properties:

(a) $\left\{x_{k}\right\}_{k=0}^{\infty}$ is bounded;

(b) $\lim _{k \rightarrow \infty}\left\|x_{k}-y_{k}\right\|=0$ and $\lim _{k \rightarrow \infty}\left\|x_{k}-T x_{k}\right\|=0$ hold;

(c) If $\left\|x_{k}-y_{k}\right\|=o\left(\lambda_{k}\right),\left\{x_{k}\right\}_{k=0}^{\infty}$ converges strongly to the unique solution of Problem 1.4. 
In this paper, we introduce and analyze a hybrid steepest-descent viscosity algorithm for solving the triple hierarchical variational inequality problem (THVIP) (for a strict pseudocontraction) with constraints of the GMEP (1.2) and the bilevel variational inequality problem (BVIP) in a real Hilbert space. The proposed algorithm is based on Korpelevich's extragradient method, Mann's iteration method, hybrid steepest-descent method (see [25]) and viscosity approximation method (see [21]) (including Halpern's iteration method). Under mild conditions, the strong convergence of the iteration sequences generated by the algorithm is derived. Our results improve and extend the corresponding results announced by some others, e.g., Iiduka [12, Theorem 4.1], Ceng et al. [2, Theorems 3.1-3.3], and Anh et al. [1, Theorem 3.1].

\section{Preliminaries}

Throughout this paper, we assume that $C$ is a nonempty closed convex subset of a real Hilbert space H. We use $\omega_{w}\left(x_{k}\right)$ to denote the weak $\omega$-limit set of the sequence $\left\{x_{k}\right\}$, i.e.,

$$
\omega_{w}\left(x_{k}\right):=\left\{x \in H: x_{k_{i}} \rightarrow x \text { for some subsequence }\left\{x_{k_{i}}\right\} \text { of }\left\{x_{k}\right\}\right\} .
$$

Recall that a mapping $\mathrm{A}: \mathrm{C} \rightarrow \mathrm{H}$ is called

(i) monotone if

$$
\langle A x-A y, x-y\rangle \geqslant 0, \quad \forall x, y \in C
$$

(ii) $\eta$-strongly monotone if there exists a constant $\eta>0$ such that

$$
\langle A x-A y, x-y\rangle \geqslant \eta\|x-y\|^{2}, \quad \forall x, y \in C ;
$$

(iii) $\alpha$-inverse-strongly monotone if there exists a constant $\alpha>0$ such that

$$
\langle A x-A y, x-y\rangle \geqslant \alpha\|A x-A y\|^{2}, \quad \forall x, y \in C .
$$

It is obvious that if $A$ is $\alpha$-inverse-strongly monotone, then $A$ is monotone and $\frac{1}{\alpha}$-Lipschitz continuous.

The metric (or nearest point) projection from $\mathrm{H}$ onto $\mathrm{C}$ is the mapping $\mathrm{P}_{\mathrm{C}}: \mathrm{H} \rightarrow \mathrm{C}$ which assigns to each point $x \in H$ the unique point $P_{C} x \in C$ satisfying the property

$$
\left\|x-P_{C} x\right\|=\inf _{y \in C}\|x-y\|=: d(x, C) .
$$

Some important properties of projections are gathered in the following proposition.

Proposition 2.1. For given $\mathrm{x} \in \mathrm{H}$ and $z \in \mathrm{C}$ :

(i) $z=\mathrm{P}_{\mathrm{C}} \mathrm{x} \Leftrightarrow\langle x-z, y-z\rangle \leqslant 0, \forall y \in C$;

(ii) $z=P_{C} x \Leftrightarrow\|x-z\|^{2} \leqslant\|x-y\|^{2}-\|y-z\|^{2}, \forall y \in C$;

(iii) $\left\langle P_{C} x-P_{C} y, x-y\right\rangle \geqslant\left\|P_{C} x-P_{C} y\right\|^{2}, \forall y \in H$.

Consequently, $\mathrm{P}_{\mathrm{C}}$ is nonexpansive and monotone.

If $A$ is an $\alpha$-inverse-strongly monotone mapping of $C$ into $H$, then it is obvious that $A$ is $\frac{1}{\alpha}$-Lipschitz continuous. We also have that, for all $u, v \in C$ and $\lambda>0$,

$$
\|(I-\lambda A) u-(I-\lambda A) v\|^{2}=\|(u-v)-\lambda(A u-A v)\|^{2} \leqslant\|u-v\|^{2}+\lambda(\lambda-2 \alpha)\|A u-A v\|^{2} .
$$

So, if $\lambda \leqslant 2 \alpha$, then $I-\lambda A$ is a nonexpansive mapping from $C$ to $H$.

Definition 2.2. A mapping $\mathrm{T}: \mathrm{H} \rightarrow \mathrm{H}$ is said to be:

(a) nonexpansive if $\|T x-T y\| \leqslant\|x-y\|$ for all $x, y \in H$; 
(b) firmly nonexpansive if $2 \mathrm{~T}-\mathrm{I}$ is nonexpansive, or equivalently, if $\mathrm{T}$ is 1-inverse strongly monotone (1-ism),

$$
\langle x-y, T x-T y\rangle \geqslant\|T x-T y\|^{2}, \quad \forall x, y \in H ;
$$

alternatively, $T$ is firmly nonexpansive if and only if $T$ can be expressed as $T=\frac{1}{2}(I+S)$, where $\mathrm{S}: \mathrm{H} \rightarrow \mathrm{H}$ is nonexpansive; projections are firmly nonexpansive.

Next we list some elementary conclusions for the MEP.

Proposition 2.3 ([8]). Assume that $\Theta: \mathrm{C} \times \mathrm{C} \rightarrow \mathbf{R}$ satisfies (A1)-(A4) and let $\varphi: \mathbf{C} \rightarrow \mathbf{R}$ be a proper lower semicontinuous and convex function. Assume that either (B1) or (B2) holds. For $\mathrm{r}>0$ and $\mathrm{x} \in \mathrm{H}$, define a mapping $\mathrm{T}_{\mathrm{r}}^{(\Theta, \varphi)}: \mathrm{H} \rightarrow \mathrm{C}$ as follows:

$$
\mathrm{T}_{\mathrm{r}}^{(\Theta, \varphi)}(\mathrm{x})=\left\{z \in \mathrm{C}: \Theta(z, y)+\varphi(\mathrm{y})-\varphi(z)+\frac{1}{\mathrm{r}}\langle\mathrm{y}-z, z-x\rangle \geqslant 0, \forall y \in C\right\},
$$

for all $\mathrm{x} \in \mathrm{H}$. Then the followings hold:

(i) for each $\mathrm{x} \in \mathrm{H}, \mathrm{T}_{\mathrm{r}}^{(\Theta, \varphi)}(\mathrm{x})$ is nonempty and single-valued;

(ii) $\mathrm{T}_{\mathrm{r}}^{(\Theta, \varphi)}$ is firmly nonexpansive, that is, for any $\mathrm{x}, \mathrm{y} \in \mathrm{H}$,

$$
\left\|T_{r}^{(\Theta, \varphi)} x-T_{r}^{(\Theta, \varphi)} y\right\|^{2} \leqslant\left\langle T_{r}^{(\Theta, \varphi)} x-T_{r}^{(\Theta, \varphi)} y, x-y\right\rangle ;
$$

(iii) $\operatorname{Fix}\left(\mathrm{T}_{\mathrm{r}}^{(\Theta, \varphi)}\right)=\operatorname{MEP}(\Theta, \varphi)$;

(iv) $\operatorname{MEP}(\Theta, \varphi)$ is closed and convex;

(v) $\left\|T_{s}^{(\Theta, \varphi)} x-T_{t}^{(\Theta, \varphi)} x\right\|^{2} \leqslant \frac{s-t}{s}\left\langle T_{s}^{(\Theta, \varphi)} x-T_{t}^{(\Theta, \varphi)} x, T_{s}^{(\Theta, \varphi)} x-x\right\rangle$ for all $s, t>0$ and $x \in H$.

We need some facts and tools in a real Hilbert space $\mathrm{H}$ which are listed as lemmas below.

Lemma 2.4. Let $X$ be a real inner product space. Then there holds the following inequality

$$
\|x+y\|^{2} \leqslant\|x\|^{2}+2\langle y, x+y\rangle, \quad \forall x, y \in X .
$$

Lemma 2.5. Let $\mathrm{A}: \mathrm{C} \rightarrow \mathrm{H}$ be a monotone mapping. In the context of the variational inequality problem the characterization of the projection (see Proposition 2.1 (i)) implies

$$
u \in \operatorname{VI}(C, A) \quad \Leftrightarrow \quad u=P_{C}(u-\lambda A u), \quad \forall \lambda>0 .
$$

Lemma 2.6 ([11, Demiclosedness principle]). Let C be a nonempty closed convex subset of a real Hilbert space $\mathrm{H}$. Let $\mathrm{S}$ be a nonexpansive self-mapping on $\mathrm{C}$ with $\operatorname{Fix}(\mathrm{S}) \neq \emptyset$. Then $\mathrm{I}-\mathrm{S}$ is demiclosed. That is, whenever $\left\{\mathrm{x}_{\mathrm{n}}\right\}$ is a sequence in $\mathrm{C}$ weakly converging to some $\mathrm{x} \in \mathrm{C}$ and the sequence $\left\{(\mathrm{I}-\mathrm{S}) \mathrm{x}_{\mathrm{n}}\right\}$ strongly converges to some $\mathrm{y}$, it follows that $(\mathrm{I}-\mathrm{S}) \mathrm{x}=\mathrm{y}$. Here $\mathrm{I}$ is the identity operator of $\mathrm{H}$.

Lemma 2.7 ([16, Proposition 2.1]). Let $\mathrm{C}$ be a nonempty closed convex subset of a real Hilbert space $\mathrm{H}$ and $\mathrm{T}: \mathrm{C} \rightarrow \mathrm{C}$ be a mapping.

(i) If $\mathrm{T}$ is a -strictly pseudocontractive mapping, then $\mathrm{T}$ satisfies the Lipschitzian condition

$$
\|T x-T y\| \leqslant \frac{1+\zeta}{1-\zeta}\|x-y\|, \quad \forall x, y \in C
$$

(ii) If $\mathrm{T}$ is a $\zeta$-strictly pseudocontractive mapping, then the mapping $\mathrm{I}-\mathrm{T}$ is semiclosed at 0 , that is, if $\left\{\mathrm{x}_{\mathrm{n}}\right\}$ is a sequence in $\mathrm{C}$ such that $\mathrm{x}_{\mathrm{n}} \rightarrow \tilde{\mathrm{x}}$ and $(\mathrm{I}-\mathrm{T}) \mathrm{x}_{\mathrm{n}} \rightarrow 0$, then $(\mathrm{I}-\mathrm{T}) \tilde{\mathrm{x}}=0$.

(iii) If $\mathrm{T}$ is $\zeta$-(quasi-)strict pseudocontraction, then the fixed-point set $\operatorname{Fix}(\mathrm{T})$ of $\mathrm{T}$ is closed and convex so that the projection $\mathrm{P}_{\mathrm{Fix}(\mathrm{T})}$ is well-defined. 
Lemma 2.8 ([26]). Let $\mathrm{C}$ be a nonempty closed convex subset of a real Hilbert space $\mathrm{H}$. Let $\mathrm{T}: \mathrm{C} \rightarrow \mathrm{C}$ be a $\zeta$-strictly pseudocontractive mapping. Let $\gamma$ and $\delta$ be two nonnegative real numbers such that $(\gamma+\delta) \zeta \leqslant \gamma$. Then

$$
\|\gamma(x-y)+\delta(T x-T y)\| \leqslant(\gamma+\delta)\|x-y\|, \quad \forall x, y \in C .
$$

The following lemma can be easily proven, and therefore, we omit the proof.

Lemma 2.9. Let $\mathrm{V}: \mathrm{H} \rightarrow \mathrm{H}$ be an l-Lipschitzian mapping and $\mathrm{F}: \mathrm{H} \rightarrow \mathrm{H}$ be an $\eta$-strongly monotone mapping. If $\mu \eta-\gamma l>0$ for $\mu, \gamma \geqslant 0$, then $\mu \mathrm{F}-\gamma \mathrm{V}$ is $(\mu \eta-\gamma \mathrm{l})$-strongly monotone, that is,

$$
\langle(\mu \mathrm{F}-\gamma \mathrm{V}) x-(\mu \mathrm{F}-\gamma \mathrm{V}) \mathrm{y}, \mathrm{x}-\mathrm{y}\rangle \geqslant(\mu \eta-\gamma \mathrm{l})\|x-y\|^{2}, \quad \forall x, y \in \mathrm{H} .
$$

Let $\mathrm{C}$ be a nonempty closed convex subset of a real Hilbert space $\mathrm{H}$. We introduce some notations. Let $\lambda$ be a number in $(0,1]$ and let $\mu>0$. Associating with a nonexpansive mapping $S: C \rightarrow H$, we define the mapping $\mathrm{S}^{\lambda}: \mathrm{C} \rightarrow \mathrm{H}$ by

$$
S^{\lambda} x:=S x-\lambda \mu \mathrm{F}(\mathrm{S} x), \quad \forall x \in C,
$$

where $\mathrm{F}: \mathrm{H} \rightarrow \mathrm{H}$ is an operator such that, for some positive constants $\kappa, \eta>0, F$ is $k$-Lipschitzian and $\eta$-strongly monotone on $\mathrm{H}$; that is, $\mathrm{F}$ satisfies the conditions:

$$
\|F x-F y\| \leqslant k\|x-y\| \text { and }\langle F x-F y, x-y\rangle \geqslant \eta\|x-y\|^{2},
$$

for all $x, y \in H$.

Lemma 2.10 ([23]). $S^{\lambda}$ is a contraction provided $0<\mu<\frac{2 \eta}{\kappa^{2}}$; that is,

$$
\left\|S^{\lambda} x-S^{\lambda} y\right\| \leqslant(1-\lambda \tau)\|x-y\|, \quad \forall x, y \in C,
$$

where $\tau=1-\sqrt{1-\mu\left(2 \eta-\mu \kappa^{2}\right)} \in(0,1]$.

Lemma 2.11 ([23]). Let $\left\{a_{n}\right\}$ be a sequence of nonnegative numbers satisfying the condition

$$
a_{n+1} \leqslant\left(1-\alpha_{n}\right) a_{n}+\alpha_{n} \beta_{n}, \quad \forall n \geqslant 0,
$$

where $\left\{\alpha_{n}\right\}$ and $\left\{\beta_{n}\right\}$ are sequences of real numbers such that

(i) $\left\{\alpha_{n}\right\} \subset[0,1]$ and $\sum_{n=0}^{\infty} \alpha_{n}=\infty$, or equivalently, $\prod_{n=0}^{\infty}\left(1-\alpha_{n}\right):=\lim _{n \rightarrow \infty} \prod_{k=0}^{n}\left(1-\alpha_{k}\right)=0$;

(ii) $\limsup _{n \rightarrow \infty} \beta_{n} \leqslant 0$, or $\sum_{n=0}^{\infty}\left|\alpha_{n} \beta_{n}\right|<\infty$.

Then, $\lim _{n \rightarrow \infty} a_{n}=0$.

Lemma 2.12 ([11]). Let $\mathrm{H}$ be a real Hilbert space. Then the followings hold:

(a) $\|x-y\|^{2}=\|x\|^{2}-\|y\|^{2}-2\langle x-y, y\rangle$ for all $x, y \in H$;

(b) $\|\lambda x+\mu y\|^{2}=\lambda\|x\|^{2}+\mu\|y\|^{2}-\lambda \mu\|x-y\|^{2}$ for all $x, y \in H$ and $\lambda, \mu \in[0,1]$ with $\lambda+\mu=1$;

(c) If $\left\{x_{\mathrm{k}}\right\}$ is a sequence in $\mathrm{H}$ such that $\mathrm{x}_{\mathrm{k}} \rightarrow \mathrm{x}$, it follows that

$$
\limsup _{k \rightarrow \infty}\left\|x_{k}-y\right\|^{2}=\limsup _{k \rightarrow \infty}\left\|x_{k}-x\right\|^{2}+\|x-y\|^{2}, \quad \forall y \in H .
$$

\section{Iterative algorithm and convergence criteria}

Let $\mathrm{C}$ be a nonempty closed convex subset of a real Hilbert space $\mathrm{H}$. In this section, we always assume the followings:

- $\mathrm{F}: \mathrm{H} \rightarrow \mathrm{H}$ is a $\mathrm{k}$-Lipschitzian and $\eta$-strongly monotone operator with positive constants $\mathrm{k}, \eta>0$; 
- $\mathrm{T}: \mathrm{H} \rightarrow \mathrm{H}$ is a $\zeta$-strictly pseudocontractive mapping and $\mathrm{V}: \mathrm{H} \rightarrow \mathrm{H}$ is an l-Lipschitzian mapping;

- $\Theta: \mathbf{C} \times \mathbf{C} \rightarrow \mathbf{R}$ is a bifunction satisfying conditions (A1)-(A4), $\varphi: \mathbf{C} \rightarrow \mathbf{R} \cup\{+\infty\}$ is a proper lower semicontinuous and convex function with restrictions (B1) or (B2), and $\mathcal{A}: \mathrm{H} \rightarrow \mathrm{H}$ is $\alpha$-inverse strongly monotone;

- $0<\mu<\frac{2 \eta}{\kappa^{2}}$ and $0 \leqslant \gamma l<\tau$ with $\tau=1-\sqrt{1-\mu\left(2 \eta-\mu \kappa^{2}\right)}$;

- $\mathrm{A}: \mathrm{C} \rightarrow \mathrm{H}$ and $\mathrm{B}: \mathrm{H} \rightarrow \mathrm{H}$ are two mappings satisfying the following hypotheses (H1)-(H4):

(H1) B is monotone on $\mathrm{H}$;

(H2) $A$ is $\beta$-inverse-strongly monotone on $\mathrm{C}$;

(H3) B is $\mathrm{L}_{2}$-Lipschitz continuous on $\mathrm{H}$;

(H4) $\operatorname{VI}(\operatorname{VI}(\Omega, \mathrm{B}), \mathrm{A}) \neq \emptyset$ where $\Omega:=\operatorname{GMEP}(\Theta, \varphi, \mathcal{A}) \cap \operatorname{Fix}(\mathrm{T})$.

Next, we introduce the following triple hierarchical variational inequality problem (THVIP) with constraints of the GMEP (1.2) and the bilevel variational inequality problem (BVIP).

Problem 3.1. The objective is to

$$
\text { find } x^{*} \in \operatorname{VI}(\operatorname{VI}(\Omega, \mathrm{B}), \mathrm{A}):=\left\{x^{*} \in \operatorname{VI}(\Omega, \mathrm{B}):\left\langle\mathrm{A} x^{*}, x-x^{*}\right\rangle \geqslant 0, \forall x \in \operatorname{VI}(\Omega, \mathrm{B})\right\} \text {. }
$$

That is, the objective is to find $x^{*} \in \operatorname{VI}(\Omega, \mathrm{B})$ such that

$$
\left\langle\mathrm{A} x^{*}, x-x^{*}\right\rangle \geqslant 0, \quad \forall x \in \operatorname{VI}(\Omega, \mathrm{B}),
$$

where $\operatorname{VI}(\Omega, \mathrm{B})$ denotes the set of solutions of the VIP: find $y^{*} \in \Omega$ such that

$$
\left\langle B y^{*}, y-y^{*}\right\rangle \geqslant 0, \quad \forall y \in \Omega \text {. }
$$

Algorithm 3.2. Initialization: choose $u \in H, x_{0} \in H, k=0,0<\lambda \leqslant 2 \beta$, positive sequences $\left\{\delta_{k}\right\},\left\{\lambda_{k}\right\}$, $\left\{\alpha_{k}\right\},\left\{\beta_{k}\right\},\left\{\gamma_{k}\right\},\left\{\bar{\epsilon}_{k}\right\}$, and $\left\{r_{k}\right\}$ such that

$$
\left\{\begin{array}{l}
\lim _{k \rightarrow \infty} \delta_{k}=0, \sum_{k=0}^{\infty} \bar{\epsilon}_{k}<\infty,\left\{r_{k}\right\} \subset[a, b] \subset(0,2 \alpha), \\
\alpha_{k}+\beta_{k}+\gamma_{k}=1 \forall k \geqslant 0, \sum_{k=0}^{\infty} \alpha_{k}=\infty, \lim _{k \rightarrow \infty}\left|r_{k+1}-r_{k}\right|=0, \\
\lim _{k \rightarrow \infty} \alpha_{k}=0, \bar{\epsilon}_{k}=o\left(\alpha_{k}\right), \lim _{k \rightarrow \infty} \beta_{k}=\xi \in\left(\zeta, \frac{1}{2}\right], \lim _{k \rightarrow \infty} \lambda_{k}=0, \lambda_{k} \leqslant \frac{1}{L_{2}} \quad \forall k \geqslant 0 .
\end{array}\right.
$$

Step 1. Compute

$$
\left\{\begin{array}{l}
\Theta\left(\mathfrak{u}_{k}, y\right)+\varphi(y)-\varphi\left(u_{k}\right)+\frac{1}{r_{k}}\left\langle y-u_{k}, u_{k}-\left(x_{k}-r_{k} \mathcal{A} x_{k}\right)\right\rangle \geqslant 0, \quad \forall y \in C, \\
\tilde{u}_{k}=\beta_{k} u_{k}+\left(1-\beta_{k}\right) T u_{k} \\
v_{k}=\alpha_{k} \gamma V x_{k}+\gamma_{k} x_{k}+\left(\left(1-\gamma_{k}\right) I-\alpha_{k} \mu F\right) \tilde{u}_{k}, \\
y_{k}:=P_{\Omega}\left(v_{k}-\lambda_{k} B v_{k}\right) \\
z_{k}:=P_{\Omega}\left(v_{k}-\lambda_{k} B y_{k}\right) .
\end{array}\right.
$$

Step 2. Inner loop $j=0,1, \ldots$ Compute

$$
\left\{\begin{array}{l}
x_{k, 0}:=z_{k}-\lambda A z_{k}, \\
y_{k, j}:=P_{\Omega}\left(x_{k, j}-\delta_{j} B x_{k, j}\right), \\
x_{k, j+1}:=\alpha_{j} x_{k, 0}+\beta_{j} x_{k, j}+\gamma_{j} P_{\Omega}\left(x_{k, j}-\delta_{j} B y_{k, j}\right) . \\
\text { If }\left\|x_{k, j+1}-P_{V I}(\Omega, B) x_{k, 0}\right\| \leqslant \bar{\epsilon}_{k} \text { then set } h_{k}:=x_{k, j+1} \text { and go to Step } 3 . \\
\text { Otherwise, increase } j \text { by } 1 \text { and repeat the inner loop Step } 2 .
\end{array}\right.
$$

Step 3. Set $x_{k+1}:=\alpha_{k} u+\beta_{k} x_{k}+\gamma_{k} h_{k}$. Then increase $k$ by 1 and go to Step 1 . 
Let $\mathrm{C}$ be a nonempty closed convex subset of $\mathrm{H}, \mathrm{B}: \mathrm{C} \rightarrow \mathrm{H}$ be monotone and $\mathrm{L}_{2}$-Lipschitz continuous on $C$, and $S: C \rightarrow C$ be a nonexpansive mapping such that $\operatorname{VI}(C, B) \cap \operatorname{Fix}(S) \neq \emptyset$. Let the sequences $\left\{x_{n}\right\}$ and $\left\{y_{n}\right\}$ be generated by

$$
\left\{\begin{array}{l}
x_{0} \in C \text { chosen arbitrarily, } \\
y_{k}=P_{C}\left(x_{k}-\delta_{k} B x_{k}\right) \\
x_{k+1}=\alpha_{k} x_{0}+\beta_{k} x_{k}+\gamma_{k} S P_{C}\left(x_{k}-\delta_{k} B y_{k}\right) \quad \forall k \geqslant 0
\end{array}\right.
$$

where $\left\{\alpha_{k}\right\},\left\{\beta_{k}\right\},\left\{\gamma_{k}\right\}$, and $\left\{\delta_{k}\right\}$ satisfy the following conditions:

$$
\left\{\begin{array}{l}
\delta_{k}>0 \quad \forall k \geqslant 0, \lim _{k \rightarrow \infty} \delta_{k}=0, \\
\alpha_{k}+\beta_{k}+\gamma_{k}=1, \quad \forall k \geqslant 0, \\
\sum_{k=0}^{\infty} \alpha_{k}=\infty, \lim _{k \rightarrow \infty} \alpha_{k}=0, \\
0<\liminf _{k \rightarrow \infty} \leqslant \limsup _{k \rightarrow \infty} \beta_{k}<1 .
\end{array}\right.
$$

Under these conditions, Yao et al. [27] proved that the sequences $\left\{x_{k}\right\}$ and $\left\{y_{k}\right\}$ converge to the same point

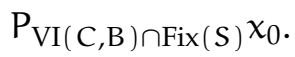

Applying these iteration sequences with $S$ being the identity mapping, we have the following lemma.

Lemma 3.3. Suppose that the hypotheses (H1)-(H4) hold. Then the sequence $\left\{x_{k, j}\right\}$ generated by Algorithm 3.2 converges strongly to the point $\mathrm{P}_{\mathrm{VI}(\Omega, \mathrm{B})}\left(z_{\mathrm{k}}-\lambda \mathrm{A} z_{\mathrm{k}}\right)$ as $\mathrm{j} \rightarrow \infty$. Consequently, we have

$$
\left\|h_{k}-P_{V I(\Omega, B)}\left(z_{k}-\lambda A z_{k}\right)\right\| \leqslant \bar{\epsilon}_{k} \quad \forall k \geqslant 0 .
$$

In the sequel we always suppose that the inner loop in Algorithm 3.2 terminates after a finite number of steps. This assumption, by Lemma 3.3, is satisfied when B is monotone on $\Omega$.

Lemma 3.4. Let sequences $\left\{v_{\mathrm{k}}\right\},\left\{\mathrm{y}_{\mathrm{k}}\right\}$, and $\left\{z_{\mathrm{k}}\right\}$ be generated by Algorithm 3.2, B be $\mathrm{L}_{2}$-Lipschitzian and monotone on $\mathrm{H}$, and $\mathrm{p} \in \mathrm{VI}(\Omega, \mathrm{B})$. Then, we have

$$
\left\|z_{k}-p\right\|^{2} \leqslant\left\|v_{k}-p\right\|^{2}-\left(1-\lambda_{k} L_{2}\right)\left\|v_{k}-y_{k}\right\|^{2}-\left(1-\lambda_{k} L_{2}\right)\left\|y_{k}-z_{k}\right\|^{2} .
$$

Proof. Let $p \in \operatorname{VI}(\Omega, B)$. This means $\langle\mathrm{B} p, x-p\rangle \geqslant 0, \forall x \in \Omega$. Then, for each $\lambda_{k}>0$, $p$ satisfies the fixed point equation $p=\mathrm{P}_{\Omega}\left(p-\lambda_{k} B p\right)$. Since $B$ is monotone on $H$ and $p \in \operatorname{VI}(\Omega$, B), we have

$$
\left\langle B y_{k}, y_{k}-p\right\rangle \geqslant\left\langle B p, y_{k}-p\right\rangle \geqslant 0 \text {. }
$$

Then, applying Proposition 2.1 (ii) with $v_{k}-\lambda_{k} B y_{k}$ and $p$, we obtain

$$
\begin{aligned}
\left\|z_{k}-p\right\|^{2} \leqslant & \left\|v_{k}-\lambda_{k} B y_{k}-p\right\|^{2}-\left\|v_{k}-\lambda_{k} B y_{k}-z_{k}\right\|^{2} \\
= & \left\|v_{k}-p\right\|^{2}-2 \lambda_{k}\left\langle B y_{k}, v_{k}-p\right\rangle+\lambda_{k}^{2}\left\|B y_{k}\right\|^{2}-\left\|v_{k}-z_{k}\right\|^{2} \\
& -\lambda_{k}^{2}\left\|B y_{k}\right\|^{2}+2 \lambda_{k}\left\langle B y_{k}, v_{k}-z_{k}\right\rangle \\
= & \left\|v_{k}-p\right\|^{2}-\left\|v_{k}-z_{k}\right\|^{2}+2 \lambda_{k}\left\langle B y_{k}, p-z_{k}\right\rangle \\
= & \left\|v_{k}-p\right\|^{2}-\left\|v_{k}-z_{k}\right\|^{2}+2 \lambda_{k}\left\langle B y_{k}, p-y_{k}\right\rangle+2 \lambda_{k}\left\langle B y_{k}, y_{k}-z_{k}\right\rangle \\
\leqslant & \left\|v_{k}-p\right\|^{2}-\left\|v_{k}-z_{k}\right\|^{2}+2 \lambda_{k}\left\langle B y_{k}, y_{k}-z_{k}\right\rangle .
\end{aligned}
$$

Applying Proposition 2.1 (i) with $v_{k}-\lambda_{k} B v_{k}$ and $z_{k}$, we also have

$$
\left\langle v_{k}-\lambda_{k} B v_{k}-y_{k}, z_{k}-y_{k}\right\rangle \leqslant 0
$$


Combining this inequality with (3.2) and observing that $B$ is $L_{2}$-Lipschitz continuous on $H$, we obtain

$$
\begin{aligned}
\left\|z_{k}-p\right\|^{2} \leqslant & \left\|v_{k}-p\right\|^{2}-\left\|\left(v_{k}-y_{k}\right)+\left(y_{k}-z_{k}\right)\right\|^{2}+2 \lambda_{k}\left\langle B y_{k}, y_{k}-z_{k}\right\rangle \\
= & \left\|v_{k}-p\right\|^{2}-\left\|v_{k}-y_{k}\right\|^{2}-\left\|y_{k}-z_{k}\right\|^{2}-2\left\langle v_{k}-y_{k}, y_{k}-z_{k}\right\rangle+2 \lambda_{k}\left\langle B y_{k}, y_{k}-z_{k}\right\rangle \\
= & \left\|v_{k}-p\right\|^{2}-\left\|v_{k}-y_{k}\right\|^{2}-\left\|y_{k}-z_{k}\right\|^{2}-2\left\langle v_{k}-\lambda_{k} B y_{k}-y_{k}, y_{k}-z_{k}\right\rangle \\
= & \left\|v_{k}-p\right\|^{2}-\left\|v_{k}-y_{k}\right\|^{2}-\left\|y_{k}-z_{k}\right\|^{2}-2\left\langle v_{k}-\lambda_{k} B v_{k}-y_{k}, y_{k}-z_{k}\right\rangle \\
& +2 \lambda_{k}\left\langle B v_{k}-B y_{k}, z_{k}-y_{k}\right\rangle \\
\leqslant & \left\|v_{k}-p\right\|^{2}-\left\|v_{k}-y_{k}\right\|^{2}-\left\|y_{k}-z_{k}\right\|^{2}+2 \lambda_{k}\left\langle B v_{k}-B y_{k}, z_{k}-y_{k}\right\rangle \\
\leqslant & \left\|v_{k}-p\right\|^{2}-\left\|v_{k}-y_{k}\right\|^{2}-\left\|y_{k}-z_{k}\right\|^{2}+2 \lambda_{k}\left\|B v_{k}-B y_{k}\right\|\left\|z_{k}-y_{k}\right\| \\
\leqslant & \left\|v_{k}-p\right\|^{2}-\left\|v_{k}-y_{k}\right\|^{2}-\left\|y_{k}-z_{k}\right\|^{2}+2 \lambda_{k} L_{2}\left\|v_{k}-y_{k}\right\|\left\|z_{k}-y_{k}\right\| \\
\leqslant & \left\|v_{k}-p\right\|^{2}-\left\|v_{k}-y_{k}\right\|^{2}-\left\|y_{k}-z_{k}\right\|^{2}+\lambda_{k} L_{2}\left(\left\|v_{k}-y_{k}\right\|^{2}+\left\|z_{k}-y_{k}\right\|^{2}\right) \\
\leqslant & \left\|v_{k}-p\right\|^{2}-\left(1-\lambda_{k} L_{2}\right)\left\|v_{k}-y_{k}\right\|^{2}-\left(1-\lambda_{k} L_{2}\right)\left\|y_{k}-z_{k}\right\|^{2} .
\end{aligned}
$$

Utilizing (2.1) and Proposition 2.3 (ii) we have $u_{k}=T_{r_{k}}^{(\Theta, \varphi)}\left(x_{k}-r_{k} \mathcal{A} x_{k}\right)$ for each $k \geqslant 0$ and hence

$$
\begin{aligned}
\left\|u_{k}-p\right\|^{2} & =\left\|T_{r_{k}}^{(\Theta, \varphi)}\left(x_{k}-r_{k} \mathcal{A} x_{k}\right)-T_{r_{k}}^{(\Theta, \varphi)}\left(p-r_{k} \mathcal{A p}\right)\right\|^{2} \\
& \leqslant\left\|\left(x_{k}-r_{k} \mathcal{A} x_{k}\right)-\left(p-r_{k} \mathcal{A p}\right)\right\|^{2} \\
& \leqslant\left\|x_{k}-p\right\|^{2}+r_{k}\left(r_{k}-2 \alpha\right)\left\|\mathcal{A} x_{k}-\mathcal{A p}\right\|^{2} \\
& \leqslant\left\|x_{k}-p\right\|^{2} .
\end{aligned}
$$

Since $\lim _{k \rightarrow \infty} \beta_{k}=\xi \in\left(\zeta, \frac{1}{2}\right]$, we may assume, without loss of generality, that $\left\{\beta_{k}\right\} \subset[c, d] \subset(\zeta, 1)$ for all $k \geqslant 0$. Putting $\sigma_{k}=1-\beta_{k}$ and $\mathcal{B}=I-T$, we know that $\mathcal{B}$ is $\frac{1-\zeta}{2}$-inverse-strongly monotone since $T$ is $\zeta$-strictly pseudocontractive. Observe that $\tilde{u}_{k}=\beta_{k} \mathfrak{u}_{k}+\left(1-\beta_{k}\right) T u_{k}=u_{k}-\sigma_{k} \mathcal{B} u_{k}$, which together with (2.1), yields

$$
\begin{aligned}
\left\|\tilde{u}_{k}-p\right\|^{2} & =\left\|u_{k}-\sigma_{k} \mathcal{B} u_{k}-\left(p-\sigma_{k} \mathcal{B} p\right)\right\|^{2} \\
& =\left\|u_{k}-p-\sigma_{k}\left(\mathcal{B} u_{k}-\mathcal{B} p\right)\right\|^{2} \\
& \leqslant\left\|u_{k}-p\right\|^{2}-\sigma_{k}\left(1-\zeta-\sigma_{k}\right)\left\|\mathcal{B} u_{k}-\mathcal{B} p\right\|^{2} \\
& =\left\|u_{k}-p\right\|^{2}-\left(1-\beta_{k}\right)\left(\beta_{k}-\zeta\right)\left\|u_{k}-T u_{k}\right\|^{2} .
\end{aligned}
$$

So, it follows from (3.4) and (3.5) that

$$
\left\|\tilde{u}_{k}-p\right\| \leqslant\left\|u_{k}-p\right\| \leqslant\left\|x_{k}-p\right\| .
$$

This completes the proof.

Lemma 3.5. Suppose that the conditions (A1)-(A4) and (H1)-(H4) hold and that the conditions (B1) or (B2) hold. Then the sequence $\left\{x_{\mathrm{k}}\right\}$ generated by Algorithm 3.2 is bounded.

Proof. Since $\lim _{k \rightarrow \infty} \alpha_{k}=0, \lim _{k \rightarrow \infty} \beta_{k}=\xi \in\left(\zeta, \frac{1}{2}\right]$ and $\alpha_{k}+\beta_{k}+\gamma_{k}=1$, we get $\lim _{k \rightarrow \infty}\left(1-\gamma_{k}\right)=$ $\lim _{k \rightarrow \infty}\left(\alpha_{k}+\beta_{k}\right)=\xi$. Hence, we may assume, without loss of generality, that $0<\frac{\alpha_{k}}{1-\gamma_{k}} \leqslant 1$ for all $k \geqslant 0$.

Take an arbitrary $p \in \operatorname{VI}(\operatorname{VI}(B, \Omega)$, A). Then we have

$$
\langle A p, x-p\rangle \geqslant 0, \quad \forall x \in \operatorname{VI}(\Omega, B),
$$

which implies $p=P_{\mathrm{VI}(\Omega, B)}(p-\lambda A p)$. Then, it follows from (2.1), Proposition 2.1 (iii), $\beta$-inverse strong monotonicity of $A$, and $0<\lambda \leqslant 2 \beta$ that

$$
\begin{aligned}
\left\|\mathrm{P}_{\mathrm{VI}(\Omega, \mathrm{B})}\left(z_{\mathrm{k}}-\lambda A z_{\mathrm{k}}\right)-\mathrm{p}\right\|^{2} & =\left\|\mathrm{P}_{\mathrm{VI}(\Omega, \mathrm{B})}\left(z_{\mathrm{k}}-\lambda A z_{\mathrm{k}}\right)-\mathrm{P}_{\mathrm{VI}(\Omega, \mathrm{B})}(\mathrm{p}-\lambda A \mathrm{p})\right\|^{2} \\
& \leqslant\left\|(\mathrm{I}-\lambda \mathrm{A}) z_{\mathrm{k}}-(\mathrm{I}-\lambda \mathrm{A}) \mathrm{p}\right\|^{2} \\
& \leqslant\left\|z_{\mathrm{k}}-\mathrm{p}\right\|^{2}+\lambda(\lambda-2 \beta)\left\|A z_{\mathrm{k}}-A \mathrm{p}\right\|^{2} \\
& \leqslant\left\|z_{\mathrm{k}}-\mathrm{p}\right\|^{2} .
\end{aligned}
$$


Furthermore, from Algorithm 3.2, Lemma 2.10, and (3.6), we have

$$
\begin{aligned}
\left\|v_{k}-p\right\|= & \left\|\alpha_{k} \gamma V x_{k}+\gamma_{k} x_{k}+\left(\left(1-\gamma_{k}\right) I-\alpha_{k} \mu F\right) \tilde{u}_{k}-p\right\| \\
= & \left\|\alpha_{k}\left(\gamma V x_{k}-\mu F p\right)+\gamma_{k}\left(x_{k}-p\right)+\left(\left(1-\gamma_{k}\right) I-\alpha_{k} \mu F\right) \tilde{u}_{k}-\left(\left(1-\gamma_{k}\right) I-\alpha_{k} \mu F\right) p\right\| \\
\leqslant & \alpha_{k} \gamma l\left\|x_{k}-p\right\|+\alpha_{k}\|(\gamma V-\mu F) p\|+\gamma_{k}\left\|x_{k}-p\right\| \\
& +\left\|\left(\left(1-\gamma_{k}\right) I-\alpha_{k} \mu F\right) \tilde{u}_{k}-\left(\left(1-\gamma_{k}\right) I-\alpha_{k} \mu F\right) p\right\| \\
\leqslant & \alpha_{k} \gamma l\left\|x_{k}-p\right\|+\alpha_{k}\|(\gamma V-\mu F) p\|+\gamma_{k}\left\|x_{k}-p\right\|+\left(1-\gamma_{k}\right)\left(1-\frac{\alpha_{k}}{1-\gamma_{k}} \tau\right)\left\|\tilde{u}_{k}-p\right\| \\
\leqslant & \alpha_{k} \gamma l\left\|x_{k}-p\right\|+\alpha_{k}\|(\gamma V-\mu F) p\|+\gamma_{k}\left\|x_{k}-p\right\|+\left(1-\gamma_{k}-\alpha_{k} \tau\right)\left\|x_{k}-p\right\| \\
= & \left(1-\alpha_{k}(\tau-\gamma l)\right)\left\|x_{k}-p\right\|+\alpha_{k}(\tau-\gamma l) \frac{\|(\gamma V-\mu F) p\|}{\tau-\gamma l} \\
\leqslant & \max \left\{\left\|x_{k}-p\right\|, \frac{\|(\gamma V-\mu F) p\|_{1}}{\tau-\gamma l}\right\} .
\end{aligned}
$$

Utilizing (3.3), (3.7), (3.8), and the assumptions $0<\lambda \leqslant 2 \beta, \quad \sum_{k=0}^{\infty} \bar{\epsilon}_{k}<\infty$ we obtain that

$$
\begin{aligned}
\left\|x_{k+1}-p\right\| & =\left\|\alpha_{k} u+\beta_{k} x_{k}+\gamma_{k} h_{k}-p\right\| \\
& \leqslant \alpha_{k}\|u-p\|+\beta_{k}\left\|x_{k}-p\right\|+\gamma_{k}\left\|h_{k}-P_{V I(\Omega, B)}\left(z_{k}-\lambda A z_{k}\right)\right\|+\gamma_{k}\left\|P_{V I(\Omega, B)}\left(z_{k}-\lambda A z_{k}\right)-p\right\| \\
& \leqslant \alpha_{k}\|u-p\|+\beta_{k}\left\|x_{k}-p\right\|+\gamma_{k} \bar{\epsilon}_{k}+\gamma_{k}\left\|z_{k}-p\right\| \\
& \leqslant \alpha_{k}\|u-p\|+\beta_{k}\left\|x_{k}-p\right\|+\gamma_{k} \bar{\epsilon}_{k}+\gamma_{k}\left\|v_{k}-p\right\| \\
& \left.\leqslant \alpha_{k}\|u-p\|+\beta_{k}\left\|x_{k}-p\right\|+\gamma_{k} \bar{\epsilon}_{k}+\gamma_{k} \max _{\{}\left\|x_{k}-p\right\|, \frac{\|(\gamma V-\mu F) p\|_{1}}{\tau-\gamma l}\right\} \\
& \leqslant \alpha_{k}\|u-p\|+\left(1-\alpha_{k}\right) \max \left\{\left\|x_{k}-p\right\|, \frac{\|(\gamma V-\mu F) p\|}{\tau-\gamma l}\right\}+\bar{\epsilon}_{k} \\
& \leqslant \max \left\{\left\|x_{k}-p\right\|,\|u-p\|, \frac{\|(\gamma V-\mu F) p\|}{\tau-\gamma l}\right\}+\bar{\epsilon}_{k} \\
& \leqslant \max \left\{\left\|x_{0}-p\right\|,\|u-p\|, \frac{\|(\gamma V-\mu F) p\|}{\tau-\gamma l}\right\}+\sum_{k=0}^{\infty} \bar{\epsilon}_{k}<\infty
\end{aligned}
$$

which shows that the sequence $\left\{x_{k}\right\}$ is bounded, and so are the sequences $\left\{u_{k}\right\},\left\{\tilde{u}_{k}\right\},\left\{v_{k}\right\},\left\{y_{k}\right\}$, and $\left\{z_{k}\right\}$.

Lemma 3.6 ([20]). Let $\left\{x_{k}\right\}$ and $\left\{y_{k}\right\}$ be two bounded sequences in a real Banach space $X$. Let $\left\{\beta_{k}\right\}$ be a sequence in $[0,1]$. Suppose that $\liminf _{k \rightarrow \infty} \beta_{k} \leqslant \limsup _{k \rightarrow \infty} \beta_{k}<1, x_{k+1}=\left(1-\beta_{k}\right) y_{k}+\beta_{k} x_{k}$ and

$$
\limsup _{k \rightarrow \infty}\left(\left\|y_{k+1}-y_{k}\right\|-\left\|x_{k+1}-x_{k}\right\|\right) \leqslant 0 \text {. }
$$

Then, $\lim _{k \rightarrow \infty}\left\|y_{k}-x_{k}\right\|=0$.

Lemma 3.7. Suppose that the conditions (A1)-(A4) and (H1)-(H4) hold. Assume that the conditions (B1) or (B2) hold and that the sequences $\left\{v_{\mathrm{k}}\right\}$ and $\left\{z_{\mathrm{k}}\right\}$ are generated by Algorithm 3.2. Then, we have

$$
\left\|z_{\mathrm{k}+1}-z_{\mathrm{k}}\right\| \leqslant\left(1+\lambda_{\mathrm{k}+1} \mathrm{~L}_{2}\right)\left\|v_{\mathrm{k}+1}-v_{\mathrm{k}}\right\|+\lambda_{\mathrm{k}}\left\|\mathrm{B} \mathrm{y}_{\mathrm{k}}\right\|+\lambda_{\mathrm{k}+1}\left(\left\|\mathrm{~B} v_{\mathrm{k}+1}\right\|+\left\|\mathrm{B} \mathrm{y}_{\mathrm{k}+1}\right\|+\left\|\mathrm{B} v_{\mathrm{k}}\right\|\right) .
$$

Moreover, $\left\{z_{\mathrm{k}}\right\}$ is bounded and $\lim _{\mathrm{k} \rightarrow \infty}\left\|z_{\mathrm{k}+1}-z_{\mathrm{k}}\right\|=\lim _{\mathrm{k} \rightarrow \infty}\left\|v_{\mathrm{k}+1}-v_{\mathrm{k}}\right\|=0$.

Proof. Since B is $L_{2}$-Lipschitzian on $H$, for each $x, y \in H$, we have

$$
\left\|\left(I-\lambda_{k} B\right) x-\left(I-\lambda_{k} B\right) y\right\| \leqslant\|x-y\|+\lambda_{k}\|B x-B y\| \leqslant\left(1+\lambda_{k} L_{2}\right)\|x-y\| .
$$


Combining this inequality with Proposition 2.1 (iii), we have

$$
\begin{aligned}
\left\|z_{k+1}-z_{k}\right\| & =\left\|P_{C}\left(v_{k+1}-\lambda_{k+1} B y_{k+1}\right)-P_{C}\left(v_{k}-\lambda_{k} B y_{k}\right)\right\| \\
& \leqslant\left\|\left(v_{k+1}-\lambda_{k+1} B y_{k+1}\right)-v_{k}+\lambda_{k} B y_{k}\right\| \\
& =\left\|\left(v_{k+1}-\lambda_{k+1} B v_{k+1}\right)-\left(v_{k}-\lambda_{k+1} B v_{k}\right)+\lambda_{k+1}\left(B v_{k+1}-B y_{k+1}-B v_{k}\right)+\lambda_{k} B y_{k}\right\| \\
& \leqslant\left(1+\lambda_{k+1} L_{2}\right)\left\|v_{k+1}-v_{k}\right\|+\lambda_{k}\left\|B y_{k}\right\|+\lambda_{k+1}\left(\left\|B v_{k+1}\right\|+\left\|B y_{k+1}\right\|+\left\|B v_{k}\right\|\right) .
\end{aligned}
$$

This is the desired result (3.9).

Now we denote $x_{k+1}=\left(1-\beta_{k}\right) w_{k}+\beta_{k} x_{k}$. Then, we have

$$
\begin{aligned}
w_{k+1}-w_{k} & =\frac{\alpha_{k+1} u+\gamma_{k+1} h_{k+1}}{1-\beta_{k+1}}-\frac{\alpha_{k} u+\gamma_{k} h_{k}}{1-\beta_{k}} \\
& =\left(\frac{\alpha_{k+1}}{1-\beta_{k+1}}-\frac{\alpha_{k}}{1-\beta_{k}}\right) u+\left(\frac{\gamma_{k+1}}{1-\beta_{k+1}}-\frac{\gamma_{k}}{1-\beta_{k}}\right) h_{k}+\frac{\gamma_{k+1}}{1-\beta_{k+1}}\left(h_{k+1}-h_{k}\right) .
\end{aligned}
$$

Note that, for $0<\lambda \leqslant 2 \beta$, we have from (2.1) that

$$
\begin{aligned}
\left\|\mathrm{P}_{\mathrm{VI}(\Omega, \mathrm{B})}\left(z_{\mathrm{k}+1}-\lambda \mathrm{A} z_{\mathrm{k}+1}\right)-\mathrm{P}_{\mathrm{VI}(\Omega, \mathrm{B})}\left(z_{\mathrm{k}}-\lambda \mathrm{A} z_{\mathrm{k}}\right)\right\|^{2} & \leqslant\left\|(\mathrm{I}-\lambda \mathrm{A}) z_{\mathrm{k}+1}-(\mathrm{I}-\lambda \mathrm{A}) z_{\mathrm{k}}\right\|^{2} \\
& \leqslant\left\|z_{\mathrm{k}+1}-z_{\mathrm{k}}\right\|^{2}+\lambda(\lambda-2 \beta)\left\|A z_{\mathrm{k}+1}-A z_{\mathrm{k}}\right\|^{2} \\
& \leqslant\left\|z_{\mathrm{k}+1}-z_{\mathrm{k}}\right\|^{2} .
\end{aligned}
$$

Then, combining (3.9) with $\lambda \leqslant 2 \beta$ and (3.10) we get

$$
\begin{aligned}
& \left\|w_{k+1}-w_{k}\right\|-\left\|x_{k+1}-x_{k}\right\| \\
& \leqslant\left|\frac{\alpha_{k+1}}{1-\beta_{k+1}}-\frac{\alpha_{k}}{1-\beta_{k}}\right|\left\|u||+\left|\frac{\gamma_{k+1}}{1-\beta_{k+1}}-\frac{\gamma_{k}}{1-\beta_{k}}\right|\right\| h_{k} \| \\
& +\frac{\gamma_{k+1}}{1-\beta_{k+1}}\left\|h_{k+1}-h_{k}\right\|-\left\|x_{k+1}-x_{k}\right\| \\
& \leqslant\left|\frac{\alpha_{k+1}}{1-\beta_{k+1}}-\frac{\alpha_{k}}{1-\beta_{k}}\right| \| u||+\left|\frac{\gamma_{k+1}}{1-\beta_{k+1}}-\frac{\gamma_{k}}{1-\beta_{k}}\right|\left(\left\|P_{V I}(\Omega, B)\left(z_{k}-\lambda A z_{k}\right)\right\|+\bar{\epsilon}_{k}\right) \\
& +\frac{\gamma_{\mathrm{k}+1}}{1-\beta_{\mathrm{k}+1}}\left\|\mathrm{P}_{\mathrm{VI}(\Omega, \mathrm{B})}\left(z_{\mathrm{k}+1}-\lambda \mathrm{A} z_{\mathrm{k}+1}\right)-\mathrm{P}_{\mathrm{VI}(\Omega, \mathrm{B})}\left(z_{\mathrm{k}}-\lambda \mathrm{A} z_{\mathrm{k}}\right)\right\| \\
& +\frac{\gamma_{k+1}}{1-\beta_{k+1}}\left(\left\|P_{\mathrm{VI}(\Omega, \mathrm{B})}\left(z_{\mathrm{k}+1}-\lambda A z_{\mathrm{k}+1}\right)-\mathrm{h}_{\mathrm{k}+1}\right\|\right. \\
& \left.+\left\|P_{\mathrm{VI}(\Omega, \mathrm{B})}\left(z_{\mathrm{k}}-\lambda A z_{\mathrm{k}}\right)-\mathrm{h}_{\mathrm{k}}\right\|\right)-\left\|\mathrm{x}_{\mathrm{k}+1}-\mathrm{x}_{\mathrm{k}}\right\| \\
& \leqslant\left|\frac{\alpha_{k+1}}{1-\beta_{k+1}}-\frac{\alpha_{k}}{1-\beta_{k}}\right|\|u\|+\left|\frac{\gamma_{k+1}}{1-\beta_{k+1}}-\frac{\gamma_{k}}{1-\beta_{k}}\right|\left(\left\|P_{V I}(\Omega, B)\left(z_{k}-\lambda A z_{k}\right)\right\|+\bar{\epsilon}_{k}\right) \\
& +\frac{\gamma_{k+1}}{1-\beta_{k+1}}\left\|z_{k+1}-z_{k}\right\|+\frac{\gamma_{k+1}}{1-\beta_{k+1}}\left(\bar{\epsilon}_{k+1}+\bar{\epsilon}_{k}\right)-\left\|x_{k+1}-x_{k}\right\| .
\end{aligned}
$$

Hence,

$$
\begin{aligned}
&\left\|w_{k+1}-w_{k}\right\|-\left\|x_{k+1}-x_{k}\right\| \\
& \leqslant\left|\frac{\alpha_{k+1}}{1-\beta_{k+1}}-\frac{\alpha_{k}}{1-\beta_{k}}\right|\|u\|+\left|\frac{\gamma_{k+1}}{1-\beta_{k+1}}-\frac{\gamma_{k}}{1-\beta_{k}}\right|\left(\left\|P_{V I}(\Omega, B)\left(z_{k}-\lambda A z_{k}\right)\right\|+\bar{\epsilon}_{k}\right) \\
&+\frac{\gamma_{k+1}\left(1+\lambda_{k+1} L_{2}\right)}{1-\beta_{k+1}}\left\|v_{k+1}-v_{k}\right\|+\frac{\gamma_{k+1}}{1-\beta_{k+1}}\left(\bar{\epsilon}_{k+1}+\bar{\epsilon}_{k}\right) \\
&+\frac{\gamma_{k+1}}{1-\beta_{k+1}}\left(\lambda_{k+1}\left(\left\|B v_{k+1}\right\|+\left\|B y_{k+1}\right\|+\left\|B v_{k}\right\|\right)+\lambda_{k}\left\|B y_{k}\right\|\right)-\left\|x_{k+1}-x_{k}\right\| \\
& \leqslant\left|\frac{\alpha_{k+1}}{1-\beta_{k+1}}-\frac{\alpha_{k}}{1-\beta_{k}}\right|\|u\|+\left|\frac{\gamma_{k+1}}{1-\beta_{k+1}}-\frac{\gamma_{k}}{1-\beta_{k}}\right|\left(\left\|P_{V I}(\Omega, B)\left(z_{k}-\lambda A z_{k}\right)\right\|+\bar{\epsilon}_{k}\right) \\
&+\frac{\gamma_{k+1}\left(1+\lambda_{k+1} L_{2}\right)}{1-\beta_{k+1}}\left\|v_{k+1}-v_{k}\right\|-\left\|x_{k+1}-x_{k}\right\|+\frac{\gamma_{k+1}}{1-\beta_{k+1}}\left(\bar{\epsilon}_{k+1}+\bar{\epsilon}_{k}\right) \\
&+\frac{\gamma_{k+1}}{1-\beta_{k+1}}\left(\lambda_{k+1}\left(\left\|B v_{k+1}\right\|+\left\|B y_{k+1}\right\|+\left\|B v_{k}\right\|\right)+\lambda_{k}\left\|B y_{k}\right\|\right) .
\end{aligned}
$$


On the other hand, utilizing Proposition 2.3 (ii), (v), we obtain

$$
\begin{aligned}
\left\|u_{k+1}-u_{k}\right\|= & \left\|T_{r_{k+1}}^{(\Theta, \varphi)}\left(I-r_{k+1} \mathcal{A}\right) x_{k+1}-T_{r_{k}}^{(\Theta, \varphi)}\left(I-r_{k} \mathcal{A}\right) x_{k}\right\| \\
\leqslant & \left\|T_{r_{k+1}}^{(\Theta, \varphi)}\left(I-r_{k+1} \mathcal{A}\right) x_{k+1}-T_{r_{k}}^{(\Theta, \varphi)}\left(I-r_{k} \mathcal{A}\right) x_{k+1}\right\| \\
& +\left\|T_{r_{k}}^{(\Theta, \varphi)}\left(I-r_{k} \mathcal{A}\right) x_{k+1}-T_{r_{k}}^{(\Theta, \varphi)}\left(I-r_{k} \mathcal{A}\right) x_{k}\right\| \\
\leqslant & \left\|T_{r_{k+1}}^{(\Theta, \varphi)}\left(I-r_{k+1} \mathcal{A}\right) x_{k+1}-T_{r_{k}}^{(\Theta, \varphi)}\left(I-r_{k+1} \mathcal{A}\right) x_{k+1}\right\| \\
& +\left\|T_{r_{k}}^{(\Theta, \varphi)}\left(I-r_{k+1} \mathcal{A}\right) x_{k+1}-T_{r_{k}}^{(\Theta, \varphi)}\left(I-r_{k} \mathcal{A}\right) x_{k+1}\right\| \\
& +\left\|\left(I-r_{k} \mathcal{A}\right) x_{k+1}-\left(I-r_{k} \mathcal{A}\right) x_{k}\right\| \\
\leqslant & \frac{\left|r_{k+1}-r_{k}\right|}{r_{k+1}}\left\|T_{r_{k+1}}^{(\Theta, \varphi)}\left(I-r_{k+1} \mathcal{A}\right) x_{k+1}-\left(I-r_{k+1} \mathcal{A}\right) x_{k+1}\right\| \\
& +\left|r_{k+1}-r_{k}\right|\left\|\mathcal{A} x_{k+1}\right\|+\left\|x_{k+1}-x_{k}\right\| \\
= & \left|r_{k+1}-r_{k}\right|\left[\left\|\mathcal{A} x_{k+1}\right\|+\frac{1}{r_{k+1}}\left\|T_{r_{k+1}}^{(\Theta, \varphi)}\left(I-r_{k+1} \mathcal{A}\right) x_{k+1}-\left(I-r_{k+1} \mathcal{A}\right) x_{k+1}\right\|\right] \\
& +\left\|x_{k+1}-x_{k}\right\| \\
\leqslant & M_{0}\left|r_{k+1}-r_{k}\right|+\left\|x_{k+1}-x_{k}\right\|
\end{aligned}
$$

where $\sup _{k \geqslant 0}\left\{\left\|\mathcal{A} x_{k+1}\right\|+\frac{1}{r_{k+1}}\left\|T_{r_{k+1}}^{(\Theta, \varphi)}\left(I-r_{k+1} \mathcal{A}\right) x_{k+1}-\left(I-r_{k+1} \mathcal{A}\right) x_{k+1}\right\|\right\} \leqslant M_{0}$ for some $M_{0}>0$. Also, utilizing Algorithm 3.2 and Lemma 2.8, we have

$$
\begin{aligned}
\left\|\tilde{u}_{k+1}-\tilde{u}_{k}\right\|= & \left\|\beta_{k+1} u_{k+1}+\left(1-\beta_{k+1}\right) T u_{k+1}-\left(\beta_{k} u_{k}+\left(1-\beta_{k}\right) T u_{k}\right)\right\| \\
= & \|\left(1-\beta_{k+1}\right)\left(T u_{k+1}-T u_{k}\right)-\left(\beta_{k+1}-\beta_{k}\right) T u_{k} \\
& +\beta_{k+1}\left(u_{k+1}-\mathfrak{u}_{k}\right)+\left(\beta_{k+1}-\beta_{k}\right) \mathfrak{u}_{k} \| \\
\leqslant & \left\|\mathfrak{u}_{k+1}-\mathfrak{u}_{k}\right\|+\left|\beta_{k+1}-\beta_{k}\right|\left\|u_{k}-T u_{k}\right\| .
\end{aligned}
$$

Moreover, we define $\tilde{w}_{k}=\frac{v_{k}-\gamma_{k} x_{k}}{1-\gamma_{k}}$, which implies that $v_{k}=\left(1-\gamma_{k}\right) \tilde{w}_{k}+\gamma_{k} x_{k}$. Simple calculations show that

$$
\begin{aligned}
\tilde{w}_{k+1}-\tilde{w}_{k} & =\frac{\nu_{k+1}-\gamma_{k+1} x_{k+1}}{1-\gamma_{k+1}}-\frac{\nu_{k}-\gamma_{k} x_{k}}{1-\gamma_{k}} \\
& =\frac{\alpha_{k+1} \gamma V x_{k+1}+\left(\left(1-\gamma_{k+1}\right) I-\alpha_{k+1} \mu F\right) \tilde{u}_{k+1}}{1-\gamma_{k+1}}-\frac{\alpha_{k} \gamma V x_{k}+\left(\left(1-\gamma_{k}\right) I-\alpha_{k} \mu F\right) \tilde{u}_{k}}{1-\gamma_{k}} \\
& =\frac{\alpha_{k+1}}{1-\gamma_{k+1}} \gamma V x_{k+1}-\frac{\alpha_{k}}{1-\gamma_{k}} \gamma V x_{k}+\tilde{u}_{k+1}-\tilde{u}_{k}+\frac{\alpha_{k}}{1-\gamma_{k}} \mu F \tilde{u}_{k}-\frac{\alpha_{k+1}}{1-\gamma_{k+1}} \mu F \tilde{u}_{k+1} \\
& =\frac{\alpha_{k+1}}{1-\gamma_{k+1}}\left(\gamma V x_{k+1}-\mu F \tilde{u}_{k+1}\right)+\frac{\alpha_{k}}{1-\gamma_{k}}\left(\mu F \tilde{u}_{k}-\gamma V x_{k}\right)+\tilde{u}_{k+1}-\tilde{u}_{k} .
\end{aligned}
$$

So, it follows from (3.12) and (3.13) that

$$
\begin{aligned}
\left\|\tilde{w}_{k+1}-\tilde{w}_{k}\right\| \leqslant & \frac{\alpha_{k+1}}{1-\gamma_{k+1}}\left(\left\|\gamma \vee x_{k+1}\right\|+\left\|\mu F \tilde{u}_{k+1}\right\|\right)+\frac{\alpha_{k}}{1-\gamma_{k}}\left(\left\|\mu F \tilde{u}_{k}\right\|+\left\|\gamma \vee x_{k}\right\|\right)+\left\|\tilde{u}_{k+1}-\tilde{u}_{k}\right\| \\
\leqslant & \frac{\alpha_{k+1}}{1-\gamma_{k+1}}\left(\left\|\gamma \vee x_{k+1}\right\|+\left\|\mu F \tilde{u}_{k+1}\right\|\right)+\frac{\alpha_{k}}{1-\gamma_{k}}\left(\left\|\mu F \tilde{u}_{k}\right\|+\left\|\gamma \vee x_{k}\right\|\right)+\left\|u_{k+1}-u_{k}\right\| \\
& +\left|\beta_{k+1}-\beta_{k}\right|\left\|u_{k}-T u_{k}\right\| \\
\leqslant & \frac{\alpha_{k+1}}{1-\gamma_{k+1}}\left(\left\|\gamma \vee x_{k+1}\right\|+\left\|\mu F \tilde{u}_{k+1}\right\|\right)+\frac{\alpha_{k}}{1-\gamma_{k}}\left(\left\|\mu F \tilde{u}_{k}\right\|+\left\|\gamma \vee x_{k}\right\|\right)+M_{0}\left|r_{k+1}-r_{k}\right| \\
& +\left\|x_{k+1}-x_{k}\right\|+\left|\beta_{k+1}-\beta_{k}\right|\left\|u_{k}-T u_{k}\right\| \\
\leqslant & \left\|x_{k+1}-x_{k}\right\|+\left(\frac{\alpha_{k+1}}{1-\gamma_{k+1}}+\frac{\alpha_{k}}{1-\gamma_{k}}+\left|r_{k+1}-r_{k}\right|+\left|\beta_{k+1}-\beta_{k}\right|\right) M_{1},
\end{aligned}
$$


where $\sup _{k \geqslant 0}\left\{\left\|\mu F \tilde{u}_{k}\right\|+\left\|\gamma V x_{k}\right\|+\left\|u_{k}-T u_{k}\right\|+M_{0}\right\} \leqslant M_{1}$ for some $M_{1}>0$. In the meantime, from $v_{k}=\left(1-\gamma_{k}\right) \tilde{w}_{k}+\gamma_{k} x_{k}$, together with (3.14), we get

$$
\begin{aligned}
\left\|v_{k+1}-v_{k}\right\|= & \left\|\gamma_{k+1} x_{k+1}+\left(1-\gamma_{k+1}\right) \tilde{w}_{k+1}-\left(\gamma_{k} x_{k}+\left(1-\gamma_{k}\right) \tilde{w}_{k}\right)\right\| \\
= & \left\|\left(1-\gamma_{k+1}\right)\left(\tilde{w}_{k+1}-\tilde{w}_{k}\right)-\left(\gamma_{k+1}-\gamma_{k}\right) \tilde{w}_{k}+\gamma_{k+1}\left(x_{k+1}-x_{k}\right)+\left(\gamma_{k+1}-\gamma_{k}\right) x_{k}\right\| \\
\leqslant & \left(1-\gamma_{k+1}\right)\left\|\tilde{w}_{k+1}-\tilde{w}_{k}\right\|+\gamma_{k+1}\left\|x_{k+1}-x_{k}\right\|+\left|\gamma_{k+1}-\gamma_{k}\right|\left\|x_{k}-\tilde{w}_{k}\right\| \\
\leqslant & \left(1-\gamma_{k+1}\right)\left[\left\|x_{k+1}-x_{k}\right\|+\left(\frac{\alpha_{k+1}}{1-\gamma_{k+1}}+\frac{\alpha_{k}}{1-\gamma_{k}}+\left|r_{k+1}-r_{k}\right|+\left|\beta_{k+1}-\beta_{k}\right|\right) M_{1}\right] \\
& +\gamma_{k+1}\left\|x_{k+1}-x_{k}\right\|+\left|\gamma_{k+1}-\gamma_{k}\right|\left\|x_{k}-\tilde{w}_{k}\right\| \\
\leqslant & \left\|x_{k+1}-x_{k}\right\|+\left(\frac{\alpha_{k+1}}{1-\gamma_{k+1}}+\frac{\alpha_{k}}{1-\gamma_{k}}+\left|r_{k+1}-r_{k}\right|+\left|\beta_{k+1}-\beta_{k}\right|\right) M_{1} \\
& +\left|\gamma_{k+1}-\gamma_{k}\right|\left\|x_{k}-\tilde{w}_{k}\right\| .
\end{aligned}
$$

Combining (3.11) and (3.15) we have

$$
\begin{aligned}
& \left\|w_{k+1}-w_{k}\right\|-\left\|x_{k+1}-x_{k}\right\| \\
& \leqslant \\
& \quad\left|\frac{\alpha_{k+1}}{1-\beta_{k+1}}-\frac{\alpha_{k}}{1-\beta_{k}}\right|\|u\|+\left|\frac{\gamma_{k+1}}{1-\beta_{k+1}}-\frac{\gamma_{k}}{1-\beta_{k}}\right|\left(\left\|P_{V I}(\Omega, B)\left(z_{k}-\lambda A z_{k}\right)\right\|+\bar{\epsilon}_{k}\right) \\
& \quad+\frac{\gamma_{k+1}\left(1+\lambda_{k+1} L_{2}\right)}{1-\beta_{k+1}}\left[\left\|x_{k+1}-x_{k}\right\|+\left(\frac{\alpha_{k+1}}{1-\gamma_{k+1}}+\frac{\alpha_{k}}{1-\gamma_{k}}+\left|r_{k+1}-r_{k}\right|+\left|\beta_{k+1}-\beta_{k}\right|\right) M_{1}\right. \\
& \left.\quad+\left|\gamma_{k+1}-\gamma_{k}\right|\left\|x_{k}-\tilde{w}_{k}\right\|\right]-\left\|x_{k+1}-x_{k}\right\|+\frac{\gamma_{k+1}}{1-\beta_{k+1}}\left(\bar{\epsilon}_{k+1}+\bar{\epsilon}_{k}\right) \\
& \quad+\frac{\gamma_{k+1}}{1-\beta_{k+1}}\left(\lambda_{k+1}\left(\left\|B v_{k+1}\right\|+\left\|B y_{k+1}\right\|+\left\|B v_{k}\right\|\right)+\lambda_{k}\left\|B y_{k}\right\|\right) \\
& =\left|\frac{\alpha_{k+1}}{1-\beta_{k+1}}-\frac{\alpha_{k}}{1-\beta_{k}}\right|\|u\|+\left|\frac{\gamma_{k+1}}{1-\beta_{k+1}}-\frac{\gamma_{k}}{1-\beta_{k}}\right|\left(\left\|P_{V I}(\Omega, B)\left(z_{k}-\lambda A z_{k}\right)\right\|+\bar{\epsilon}_{k}\right) \\
& \quad+\frac{\gamma_{k+1}\left(1+\lambda_{k+1} L_{2}\right)}{1-\beta_{k+1}}\left[\left(\frac{\alpha_{k+1}}{1-\gamma_{k+1}}+\frac{\alpha_{k}}{1-\gamma_{k}}+\left|r_{k+1}-r_{k}\right|+\left|\beta_{k+1}-\beta_{k}\right|\right) M_{1}\right. \\
& \left.\quad+\left|\gamma_{k+1}-\gamma_{k}\right|\left\|x_{k}-\tilde{w}_{k}\right\|\right]+\left(\frac{\gamma_{k+1}\left(1+\lambda_{k+1} L_{2}\right)}{1-\beta_{k+1}}-1\right)\left\|x_{k+1}-x_{k}\right\|+\frac{\gamma_{k+1}}{1-\beta_{k+1}}\left(\bar{\epsilon}_{k+1}+\bar{\epsilon}_{k}\right) \\
& \quad+\frac{\gamma_{k+1}}{1-\beta_{k+1}}\left(\lambda_{k+1}\left(\left\|B v_{k+1}\right\|+\left\|B y_{k+1}\right\|+\left\|B v_{k}\right\|\right)+\lambda_{k}\left\|B y_{k}\right\|\right) .
\end{aligned}
$$

From the assumptions $\alpha_{k}+\beta_{k}+\gamma_{k}=1, \lim _{k \rightarrow \infty} \beta_{k}=\xi \in\left(\zeta, \frac{1}{2}\right], \lim _{k \rightarrow \infty} \alpha_{k}=0$, and $\lim _{k \rightarrow \infty} \lambda_{k}=0$, it follows that $\lim _{k \rightarrow \infty}\left|\beta_{k+1}-\beta_{k}\right|=\lim _{k \rightarrow \infty}\left|\gamma_{k+1}-\gamma_{k}\right|=0$,

$$
\lim _{k \rightarrow \infty}\left|\frac{\alpha_{k+1}}{1-\beta_{k+1}}-\frac{\alpha_{k}}{1-\beta_{k}}\right|=\lim _{k \rightarrow \infty}\left|\frac{\gamma_{k+1}}{1-\beta_{k+1}}-\frac{\gamma_{k}}{1-\beta_{k}}\right|=0 \quad \text { and } \quad \lim _{k \rightarrow \infty} \frac{\gamma_{k+1}\left(1+\lambda_{k+1} L_{2}\right)}{1-\beta_{k+1}}=1 .
$$

Combining these equalities with (3.16), we obtain from Lemma 3.5, $\lim _{k \rightarrow \infty} \bar{\epsilon}_{k}=0$, and $\lim _{k \rightarrow \infty} \mid r_{k+1}-$ $r_{k} \mid=0$ that

$$
\limsup _{k \rightarrow \infty}\left(\left\|w_{k+1}-w_{k}\right\|-\left\|x_{k+1}-x_{k}\right\|\right) \leqslant 0
$$

Now applying Lemma 3.6, we have

$$
\lim _{k \rightarrow \infty}\left\|w_{k}-x_{k}\right\|=0
$$

Hence by $x_{k+1}=\left(1-\beta_{k}\right) w_{k}+\beta_{k} x_{k}$, we deduce that

$$
\lim _{k \rightarrow \infty}\left\|x_{k+1}-x_{k}\right\|=\lim _{k \rightarrow \infty}\left(1-\beta_{k}\right)\left\|w_{k}-x_{k}\right\|=0,
$$

which together with $\lim _{k \rightarrow \infty} \lambda_{k}=0$, (3.9), and (3.15), implies that

$$
\lim _{k \rightarrow \infty}\left\|v_{k+1}-v_{k}\right\|=0 \quad \text { and } \quad \lim _{k \rightarrow \infty}\left\|z_{k+1}-z_{k}\right\|=0 \text {. }
$$


Lemma 3.8. Suppose that the conditions (A1)-(A4) and (H1)-(H4) hold and that the conditions (B1) or (B2) hold. Then for any $\mathrm{p} \in \operatorname{VI}(\operatorname{VI}(\Omega, \mathrm{B}), \mathrm{A})$ we have

$$
\begin{aligned}
\left\|x_{k+1}-p\right\|^{2} \leqslant & \alpha_{k}\|u-p\|^{2}+\beta_{k}\left\|x_{k}-p\right\|^{2}+\gamma_{k}\left\|v_{k}-p\right\|^{2}+2 \gamma_{k} \bar{\epsilon}_{k}\left\|z_{k}-p\right\| \\
& +\gamma_{k} \bar{\epsilon}_{k}^{2}-\gamma_{k}\left(1-\lambda_{k} L_{2}\right)\left(\left\|v_{k}-y_{k}\right\|^{2}+\left\|y_{k}-z_{k}\right\|^{2}\right) .
\end{aligned}
$$

Moreover

$$
\lim _{k \rightarrow \infty}\left\|P_{\mathrm{VI}(\Omega, B)}\left(z_{k}-\lambda_{k} A z_{k}\right)-z_{k}\right\|=\lim _{k \rightarrow \infty}\left\|P_{V I(\Omega, B)}\left(y_{k}-\lambda_{k} A y_{k}\right)-y_{k}\right\|=0 .
$$

Proof. By Lemma 3.3, we know that $\lim _{j \rightarrow \infty} x_{k, j}=P_{\mathrm{VI}(\Omega, B}\left(z_{k}-\lambda A z_{k}\right)$ which together with $0<\lambda \leqslant 2 \beta$, inequality (3.1), $\lim _{k \rightarrow \infty} \beta_{\mathrm{k}}=\xi \in\left(\zeta, \frac{1}{2}\right]$, and $p \in \operatorname{VI}(\mathrm{VI}(\Omega, \mathrm{B}), \mathrm{A})$, implies that

$$
\begin{aligned}
\left\|x_{k+1}-p\right\|^{2}= & \left\|\alpha_{k} u+\beta_{k} x_{k}+\gamma_{k} h_{k}-p\right\|^{2} \\
\leqslant & \alpha_{k}\|u-p\|^{2}+\beta_{k}\left\|x_{k}-p\right\|^{2}+\gamma_{k}\left\|h_{k}-p\right\|^{2} \\
\leqslant & \alpha_{k}\|u-p\|^{2}+\beta_{k}\left\|x_{k}-p\right\|^{2}+\gamma_{k}\left(\left\|P_{V I}(\Omega, B)\left(z_{k}-\lambda A z_{k}\right)-p\right\|+\bar{\epsilon}_{k}\right)^{2} \\
= & \alpha_{k}\|u-p\|^{2}+\beta_{k}\left\|x_{k}-p\right\|^{2} \\
& +\gamma_{k}\left(\left\|P_{V I}(\Omega, B)\left(z_{k}-\lambda A z_{k}\right)-P_{V I}(\Omega, B)(p-\lambda A p)\right\|+\bar{\epsilon}_{k}\right)^{2} \\
\leqslant & \alpha_{k}\|u-p\|^{2}+\beta_{k}\left\|x_{k}-p\right\|^{2}+\gamma_{k}\left(\left\|(I-\lambda A) z_{k}-(I-\lambda A) p\right\|+\bar{\epsilon}_{k}\right)^{2} \\
\leqslant & \alpha_{k}\|u-p\|^{2}+\beta_{k}\left\|x_{k}-p\right\|^{2}+\gamma_{k}\left(\left\|z_{k}-p\right\|+\bar{\epsilon}_{k}\right)^{2} \\
= & \alpha_{k}\|u-p\|^{2}+\beta_{k}\left\|x_{k}-p\right\|^{2}+\gamma_{k}\left\|z_{k}-p\right\|^{2}+2 \gamma_{k} \bar{\epsilon}_{k}\left\|z_{k}-p\right\|+\gamma_{k} \bar{\epsilon}_{k}^{2} \\
\leqslant & \alpha_{k}\|u-p\|^{2}+\beta_{k}\left\|x_{k}-p\right\|^{2}+2 \gamma_{k} \bar{\epsilon}_{k}\left\|z_{k}-p\right\|+\gamma_{k} \bar{\epsilon}_{k}^{2} \\
& +\gamma_{k}\left(\left\|v_{k}-p\right\|^{2}-\left(1-\lambda_{k} L_{2}\right)\left\|v_{k}-y_{k}\right\|^{2}-\left(1-\lambda_{k} L_{2}\right)\left\|y_{k}-z_{k}\right\|^{2}\right) \\
= & \alpha_{k}\|u-p\|^{2}+\beta_{k}\left\|x_{k}-p\right\|^{2}+\gamma_{k}\left\|v_{k}-p\right\|^{2}+2 \gamma_{k} \bar{\epsilon}_{k}\left\|z_{k}-p\right\|+\gamma_{k} \bar{\epsilon}_{k}^{2} \\
& -\gamma_{k}\left(1-\lambda_{k} L_{2}\right)\left(\left\|v_{k}-y_{k}\right\|^{2}+\left\|y_{k}-z_{k}\right\|^{2}\right) .
\end{aligned}
$$

On the other hand, from Algorithm 3.2 we have

$$
\begin{aligned}
\left\|v_{k}-p\right\|^{2}= & \left\|\alpha_{k} \gamma V x_{k}+\gamma_{k} x_{k}+\left(\left(1-\gamma_{k}\right) I-\alpha_{k} \mu F\right) \tilde{u}_{k}-p\right\|^{2} \\
= & \left\|\alpha_{k}\left(\gamma V x_{k}-\mu F p\right)+\gamma_{k}\left(x_{k}-p\right)+\left(\left(1-\gamma_{k}\right) I-\alpha_{k} \mu F\right) \tilde{u}_{k}-\left(\left(1-\gamma_{k}\right) I-\alpha_{k} \mu F\right) p\right\|^{2} \\
= & \| \alpha_{k} \gamma\left(V x_{k}-V p\right)+\alpha_{k}(\gamma V p-\mu F p)+\gamma_{k}\left(x_{k}-p\right) \\
& +\left(\left(1-\gamma_{k}\right) I-\alpha_{k} \mu F\right) \tilde{u}_{k}-\left(\left(1-\gamma_{k}\right) I-\alpha_{k} \mu F\right) p \|^{2} \\
\leqslant & \| \alpha_{k} \gamma\left(V x_{k}-V p\right)+\gamma_{k}\left(x_{k}-p\right)+\left(\left(1-\gamma_{k}\right) I-\alpha_{k} \mu F\right) \tilde{u}_{k} \\
& -\left(\left(1-\gamma_{k}\right) I-\alpha_{k} \mu F\right) p \|^{2}+2 \alpha_{k}\left\langle(\gamma V-\mu F) p, v_{k}-p\right\rangle \\
\leqslant & {\left[\alpha_{k} \gamma\left\|V x_{k}-V p\right\|+\gamma_{k}\left\|x_{k}-p\right\|+\|\left(\left(1-\gamma_{k}\right) I-\alpha_{k} \mu F\right) \tilde{u}_{k}\right.} \\
& \left.-\left(\left(1-\gamma_{k}\right) I-\alpha_{k} \mu F\right) p \|\right]^{2}+2 \alpha_{k}\left\langle(\gamma V-\mu F) p, v_{k}-p\right\rangle \\
\leqslant & {\left[\alpha_{k} \gamma l\left\|x_{k}-p\right\|+\gamma_{k}\left\|x_{k}-p\right\|+\left(1-\gamma_{k}-\alpha_{k} \tau\right)\left\|\tilde{u}_{k}-p\right\|\right]^{2}+2 \alpha_{k}\left\langle(\gamma V-\mu F) p, v_{k}-p\right\rangle } \\
= & {\left[\alpha_{k} \tau \frac{\gamma l}{\tau}\left\|x_{k}-p\right\|+\gamma_{k}\left\|x_{k}-p\right\|+\left(1-\gamma_{k}-\alpha_{k} \tau\right)\left\|\tilde{u}_{k}-p\right\|\right]^{2}+2 \alpha_{k}\left\langle(\gamma V-\mu F) p, v_{k}-p\right\rangle } \\
\leqslant & \alpha_{k} \tau \frac{(\gamma l)^{2}}{\tau^{2}}\left\|x_{k}-p\right\|^{2}+\gamma_{k}\left\|x_{k}-p\right\|^{2}+\left(1-\gamma_{k}-\alpha_{k} \tau\right)\left\|\tilde{u}_{k}-p\right\|^{2}+2 \alpha_{k}\left\langle(\gamma V-\mu F) p, v_{k}-p\right\rangle \\
\leqslant & \alpha_{k} \frac{(\gamma l)^{2}}{\tau}\left\|x_{k}-p\right\|^{2}+\gamma_{k}\left\|x_{k}-p\right\|^{2}+\left(1-\gamma_{k}-\alpha_{k} \tau\right)\left[\left\|u_{k}-p\right\|^{2}\right. \\
& \left.-\left(1-\beta_{k}\right)\left(\beta_{k}-\zeta\right)\left\|u_{k}-T u_{k}\right\|^{2}\right]+2 \alpha_{k}\left\langle(\gamma V-\mu F) p, v_{k}-p\right\rangle \\
\leqslant & \alpha_{k} \frac{(\gamma l)^{2}}{\tau}\left\|x_{k}-p\right\|^{2}+\gamma_{k}\left\|x_{k}-p\right\|^{2}+\left(1-\gamma_{k}-\alpha_{k} \tau\right)\left[\left\|x_{k}-p\right\|^{2}+r_{k}\left(r_{k}-2 \alpha\right)\left\|\mathcal{A} x_{k}-\mathcal{A} p\right\|^{2}\right.
\end{aligned}
$$




$$
\left.-\left(1-\beta_{k}\right)\left(\beta_{k}-\zeta\right)\left\|u_{k}-T u_{k}\right\|^{2}\right]+2 \alpha_{k}\left\langle(\gamma V-\mu F) p, v_{k}-p\right\rangle
$$

Thus,

$$
\begin{aligned}
\left\|v_{k}-p\right\|^{2} \leqslant & \left(1-\alpha_{k} \frac{\tau^{2}-(\gamma l)^{2}}{\tau}\right)\left\|x_{k}-p\right\|^{2}-\left(1-\gamma_{k}-\alpha_{k} \tau\right)\left[r_{k}\left(2 \alpha-r_{k}\right)\left\|\mathcal{A} x_{k}-\mathcal{A p}\right\|^{2}\right. \\
& \left.+\left(1-\beta_{k}\right)\left(\beta_{k}-\zeta\right)\left\|u_{k}-T u_{k}\right\|^{2}\right]+2 \alpha_{k}\left\langle(\gamma V-\mu F) p, v_{k}-p\right\rangle \\
\leqslant & \left\|x_{k}-p\right\|^{2}-\left(1-\gamma_{k}-\alpha_{k} \tau\right)\left[r_{k}\left(2 \alpha-r_{k}\right)\left\|\mathcal{A} x_{k}-\mathcal{A p}\right\|^{2}\right. \\
& \left.+\left(1-\beta_{k}\right)\left(\beta_{k}-\zeta\right)\left\|u_{k}-T u_{k}\right\|^{2}\right]+2 \alpha_{k}\left\langle(\gamma V-\mu F) p, v_{k}-p\right\rangle .
\end{aligned}
$$

Combining (3.19) and (3.20), we get

$$
\begin{aligned}
\left\|x_{k+1}-p\right\|^{2} \leqslant & \alpha_{k}\|u-p\|^{2}+\beta_{k}\left\|x_{k}-p\right\|^{2}+\gamma_{k}\left\|v_{k}-p\right\|^{2}+2 \gamma_{k} \bar{\epsilon}_{k}\left\|z_{k}-p\right\| \\
& +\gamma_{k} \bar{\epsilon}_{k}^{2}-\gamma_{k}\left(1-\lambda_{k} L_{2}\right)\left(\left\|v_{k}-y_{k}\right\|^{2}+\left\|y_{k}-z_{k}\right\|^{2}\right) \\
\leqslant & \alpha_{k}\|u-p\|^{2}+\beta_{k}\left\|x_{k}-p\right\|^{2}+\gamma_{k}\left\{\left\|x_{k}-p\right\|^{2}\right. \\
& -\left(1-\gamma_{k}-\alpha_{k} \tau\right)\left[r_{k}\left(2 \alpha-r_{k}\right)\left\|\mathcal{A} x_{k}-\mathcal{A} p\right\|^{2}+\left(1-\beta_{k}\right)\left(\beta_{k}-\zeta\right)\left\|u_{k}-T u_{k}\right\|^{2}\right] \\
& \left.+2 \alpha_{k}\left\langle(\gamma V-\mu F) p, v_{k}-p\right\rangle\right\}+2 \gamma_{k} \bar{\epsilon}_{k}\left\|z_{k}-p\right\| \\
& +\gamma_{k} \bar{\epsilon}_{k}^{2}-\gamma_{k}\left(1-\lambda_{k} L_{2}\right)\left(\left\|v_{k}-y_{k}\right\|^{2}+\left\|y_{k}-z_{k}\right\|^{2}\right) \\
\leqslant & \alpha_{k}\|u-p\|^{2}+\left\|x_{k}-p\right\|^{2}-\gamma_{k}\left(1-\gamma_{k}-\alpha_{k} \tau\right)\left[r_{k}\left(2 \alpha-r_{k}\right)\left\|\mathcal{A} x_{k}-\mathcal{A} p\right\|^{2}\right. \\
& \left.+\left(1-\beta_{k}\right)\left(\beta_{k}-\zeta\right)\left\|u_{k}-T u_{k}\right\|^{2}\right]+2 \alpha_{k}\|(\gamma V-\mu F) p\|\left\|v_{k}-p\right\| \\
& +2 \gamma_{k} \bar{\epsilon}_{k}\left\|z_{k}-p\right\|+\gamma_{k} \bar{\epsilon}_{k}^{2}-\gamma_{k}\left(1-\lambda_{k} L_{2}\right)\left(\left\|v_{k}-y_{k}\right\|^{2}+\left\|y_{k}-z_{k}\right\|^{2}\right),
\end{aligned}
$$

which immediately yields

$$
\begin{aligned}
& \gamma_{k}\left(1-\gamma_{k}-\alpha_{k} \tau\right)\left[r_{k}\left(2 \alpha-r_{k}\right)\left\|\mathcal{A} x_{k}-\mathcal{A} p\right\|^{2}+\left(1-\beta_{k}\right)\left(\beta_{k}-\zeta\right)\left\|u_{k}-T u_{k}\right\|^{2}\right] \\
& \quad+\gamma_{k}\left(1-\lambda_{k} L_{2}\right)\left(\left\|v_{k}-y_{k}\right\|^{2}+\left\|y_{k}-z_{k}\right\|^{2}\right) \\
& \leqslant \\
& \alpha_{k}\|u-p\|^{2}+\left\|x_{k}-p\right\|^{2}-\left\|x_{k+1}-p\right\|^{2}+2 \alpha_{k}\|(\gamma V-\mu F) p\|\left\|v_{k}-p\right\|+2 \gamma_{k} \bar{\epsilon}_{k}\left\|z_{k}-p\right\|+\gamma_{k} \bar{\epsilon}_{k}^{2} \\
& \leqslant \\
& \quad \alpha_{k}\|u-p\|^{2}+\left\|x_{k}-x_{k+1}\right\|\left(\left\|x_{k}-p\right\|+\left\|x_{k+1}-p\right\|\right)+2 \alpha_{k}\|(\gamma V-\mu F) p\|\left\|v_{k}-p\right\| \\
& \quad+2 \gamma_{k} \bar{\epsilon}_{k}\left\|z_{k}-p\right\|+\gamma_{k} \bar{\epsilon}_{k}^{2} .
\end{aligned}
$$

Since $\alpha_{k}+\beta_{k}+\gamma_{k}=1, \alpha_{k} \rightarrow 0, \beta_{k} \rightarrow \xi \in\left(\zeta, \frac{1}{2}\right], \bar{\epsilon}_{k} \rightarrow 0, \lambda_{k} \rightarrow 0,\left\|x_{k}-x_{k+1}\right\| \rightarrow 0$, and $\left\{r_{k}\right\} \subset[a, b] \subset$ $(0,2 \alpha)$, from the boundedness of $\left\{x_{k}\right\},\left\{v_{k}\right\}$, and $\left\{z_{k}\right\}$ we obtain

$$
\lim _{k \rightarrow \infty}\left\|\mathcal{A} x_{k}-\mathcal{A p}\right\|=\lim _{k \rightarrow \infty}\left\|u_{k}-T u_{k}\right\|=\lim _{k \rightarrow \infty}\left\|v_{k}-y_{k}\right\|=\lim _{k \rightarrow \infty}\left\|y_{k}-z_{k}\right\|=0
$$

Also, utilizing Proposition 2.3 (ii) and Lemma 2.12 (a), we obtain from (2.1) and $\left\{r_{k}\right\} \subset[a, b] \subset(0,2 \alpha)$ that

$$
\begin{aligned}
\left\|u_{k}-p\right\|^{2} & =\left\|T_{r_{k}}^{(\Theta, \varphi)}\left(I-r_{k} \mathcal{A}\right) x_{k}-T_{r_{k}}^{(\Theta, \varphi)}\left(I-r_{k} \mathcal{A}\right) p\right\|^{2} \\
& \leqslant\left\langle\left(I-r_{k} \mathcal{A}\right) x_{k}-\left(I-r_{k} \mathcal{A}\right) p, u_{k}-p\right\rangle \\
& =\frac{1}{2}\left(\left\|\left(I-r_{k} \mathcal{A}\right) x_{k}-\left(I-r_{k} \mathcal{A}\right) p\right\|^{2}+\left\|u_{k}-p\right\|^{2}-\left\|\left(I-r_{k} \mathcal{A}\right) x_{k}-\left(I-r_{k} \mathcal{A}\right) p-\left(u_{k}-p\right)\right\|^{2}\right) \\
& \leqslant \frac{1}{2}\left(\left\|x_{k}-p\right\|^{2}+\left\|u_{k}-p\right\|^{2}-\left\|\left(I-r_{k} \mathcal{A}\right) x_{k}-\left(I-r_{k} \mathcal{A}\right) p-\left(u_{k}-p\right)\right\|^{2}\right) \\
& =\frac{1}{2}\left(\left\|x_{k}-p\right\|^{2}+\left\|u_{k}-p\right\|^{2}-\left\|x_{k}-u_{k}-r_{k}\left(\mathcal{A} x_{k}-\mathcal{A} p\right)\right\|^{2}\right),
\end{aligned}
$$

which immediately leads to

$$
\begin{aligned}
\left\|u_{k}-p\right\|^{2} & \leqslant\left\|x_{k}-p\right\|^{2}-\left\|x_{k}-u_{k}-r_{k}\left(\mathcal{A} x_{k}-\mathcal{A p}\right)\right\|^{2} \\
& =\left\|x_{k}-p\right\|^{2}-\left\|x_{k}-u_{k}\right\|^{2}-r_{k}^{2}\left\|\mathcal{A} x_{k}-\mathcal{A p}\right\|^{2}+2 r_{k}\left\langle\mathcal{A} x_{k}-\mathcal{A} p, x_{k}-u_{k}\right\rangle \\
& \leqslant\left\|x_{k}-p\right\|^{2}-\left\|x_{k}-u_{k}\right\|^{2}+2 r_{k}\left\|\mathcal{A} x_{k}-\mathcal{A p}\right\|\left\|x_{k}-u_{k}\right\| .
\end{aligned}
$$


Combining (3.20) and (3.22) we have

$$
\begin{aligned}
\left\|v_{k}-p\right\|^{2} \leqslant & \alpha_{k} \frac{(\gamma l)^{2}}{\tau}\left\|x_{k}-p\right\|^{2}+\gamma_{k}\left\|x_{k}-p\right\|^{2}+\left(1-\gamma_{k}-\alpha_{k} \tau\right)\left[\left\|u_{k}-p\right\|^{2}\right. \\
& \left.-\left(1-\beta_{k}\right)\left(\beta_{k}-\zeta\right)\left\|u_{k}-T u_{k}\right\|^{2}\right]+2 \alpha_{k}\left\langle(\gamma V-\mu F) p, v_{k}-p\right\rangle \\
\leqslant & \alpha_{k} \frac{(\gamma l)^{2}}{\tau}\left\|x_{k}-p\right\|^{2}+\gamma_{k}\left\|x_{k}-p\right\|^{2}+\left(1-\gamma_{k}-\alpha_{k} \tau\right)\left\|u_{k}-p\right\|^{2} \\
& +2 \alpha_{k}\left\langle(\gamma V-\mu F) p, v_{k}-p\right\rangle \\
\leqslant & \alpha_{k} \frac{(\gamma l)^{2}}{\tau}\left\|x_{k}-p\right\|^{2}+\gamma_{k}\left\|x_{k}-p\right\|^{2}+\left(1-\gamma_{k}-\alpha_{k} \tau\right)\left[\left\|x_{k}-p\right\|^{2}\right. \\
& \left.-\left\|x_{k}-u_{k}\right\|^{2}+2 r_{k}\left\|\mathcal{A} x_{k}-\mathcal{A p}\right\|\left\|x_{k}-u_{k}\right\|\right]+2 \alpha_{k}\left\langle(\gamma V-\mu F) p, v_{k}-p\right\rangle \\
\leqslant & \left(1-\alpha_{k} \frac{\tau^{2}-(\gamma l)^{2}}{\tau}\right)\left\|x_{k}-p\right\|^{2}-\left(1-\gamma_{k}-\alpha_{k} \tau\right)\left\|x_{k}-u_{k}\right\|^{2} \\
& +2 r_{k}\left\|\mathcal{A} x_{k}-\mathcal{A p}\right\|\left\|x_{k}-u_{k}\right\|+2 \alpha_{k}\|(\gamma V-\mu F) p\|\left\|v_{k}-p\right\| \\
\leqslant & \left\|x_{k}-p\right\|^{2}-\left(1-\gamma_{k}-\alpha_{k} \tau\right)\left\|x_{k}-u_{k}\right\|^{2}+2 r_{k}\left\|\mathcal{A} x_{k}-\mathcal{A p}\right\|\left\|x_{k}-u_{k}\right\| \\
& +2 \alpha_{k}\|(\gamma V-\mu F) p\|\left\|v_{k}-p\right\|,
\end{aligned}
$$

which together with (3.18), implies that

$$
\begin{aligned}
\left\|x_{k+1}-p\right\|^{2} \leqslant & \alpha_{k}\|u-p\|^{2}+\beta_{k}\left\|x_{k}-p\right\|^{2}+\gamma_{k}\left\|v_{k}-p\right\|^{2}+2 \gamma_{k} \bar{\epsilon}_{k}\left\|z_{k}-p\right\| \\
& +\gamma_{k} \bar{\epsilon}_{k}^{2}-\gamma_{k}\left(1-\lambda_{k} L_{2}\right)\left(\left\|v_{k}-y_{k}\right\|^{2}+\left\|y_{k}-z_{k}\right\|^{2}\right) \\
\leqslant & \alpha_{k}\|u-p\|^{2}+\beta_{k}\left\|x_{k}-p\right\|^{2}+\gamma_{k}\left\|v_{k}-p\right\|^{2}+2 \gamma_{k} \bar{\epsilon}_{k}\left\|z_{k}-p\right\|+\gamma_{k} \bar{\epsilon}_{k}^{2} \\
\leqslant & \alpha_{k}\|u-p\|^{2}+\beta_{k}\left\|x_{k}-p\right\|^{2}+\gamma_{k}\left[\left\|x_{k}-p\right\|^{2}-\left(1-\gamma_{k}-\alpha_{k} \tau\right)\left\|x_{k}-u_{k}\right\|^{2}\right. \\
& \left.+2 r_{k}\left\|\mathcal{A} x_{k}-\mathcal{A} p\right\|\left\|x_{k}-u_{k}\right\|+2 \alpha_{k}\|(\gamma V-\mu F) p\|\left\|v_{k}-p\right\|\right]+2 \gamma_{k} \bar{\epsilon}_{k}\left\|z_{k}-p\right\|+\gamma_{k} \bar{\epsilon}_{k}^{2} \\
\leqslant & \alpha_{k}\|u-p\|^{2}+\left\|x_{k}-p\right\|^{2}-\gamma_{k}\left(1-\gamma_{k}-\alpha_{k} \tau\right)\left\|x_{k}-u_{k}\right\|^{2} \\
& +2 r_{k}\left\|\mathcal{A} x_{k}-\mathcal{A} p\right\|\left\|x_{k}-u_{k}\right\|+2 \alpha_{k}\|(\gamma V-\mu F) p\|\left\|v_{k}-p\right\|+2 \gamma_{k} \bar{\epsilon}_{k}\left\|z_{k}-p\right\|+\gamma_{k} \bar{\epsilon}_{k}^{2} .
\end{aligned}
$$

So, it follows that

$$
\begin{aligned}
\gamma_{k}\left(1-\gamma_{k}-\alpha_{k} \tau\right)\left\|x_{k}-u_{k}\right\|^{2} \leqslant & \alpha_{k}\|u-p\|^{2}+\left\|x_{k}-p\right\|^{2}-\left\|x_{k+1}-p\right\|^{2}+2 r_{k}\left\|\mathcal{A} x_{k}-\mathcal{A p}\right\|\left\|x_{k}-u_{k}\right\| \\
& +2 \alpha_{k}\|(\gamma V-\mu F) p\|\left\|v_{k}-p\right\|+2 \gamma_{k} \bar{\epsilon}_{k}\left\|z_{k}-p\right\|+\gamma_{k} \bar{\epsilon}_{k}^{2} \\
\leqslant & \alpha_{k}\|u-p\|^{2}+\left\|x_{k}-x_{k+1}\right\|\left(\left\|x_{k}-p\right\|+\left\|x_{k+1}-p\right\|\right)+2 r_{k}\left\|\mathcal{A} x_{k}-\mathcal{A} p\right\| \\
& \times\left\|x_{k}-u_{k}\right\|+2 \alpha_{k}\|(\gamma V-\mu F) p\|\left\|v_{k}-p\right\|+2 \gamma_{k} \bar{\epsilon}_{k}\left\|z_{k}-p\right\|+\gamma_{k} \bar{\epsilon}_{k}^{2} .
\end{aligned}
$$

Since $\alpha_{k}+\beta_{k}+\gamma_{k}=1, \alpha_{k} \rightarrow 0, \beta_{k} \rightarrow \xi \in\left(\zeta, \frac{1}{2}\right], \bar{\epsilon}_{k} \rightarrow 0,\left\|x_{k}-x_{k+1}\right\| \rightarrow 0,\left\|\mathcal{A} x_{k}-\mathcal{A p}\right\| \rightarrow 0$, and $\left\{r_{k}\right\} \subset[a, b] \subset(0,2 \alpha)$, from the boundedness of $\left\{x_{k}\right\},\left\{u_{k}\right\},\left\{v_{k}\right\}$, and $\left\{z_{k}\right\}$ we obtain

$$
\lim _{k \rightarrow \infty}\left\|x_{k}-u_{k}\right\|=0 \text {. }
$$

Thus, from $\alpha_{k} \rightarrow 0$, Algorithm 3.2, (3.21), and (3.23) it follows that as $k \rightarrow \infty$,

$$
\left\|\tilde{u}_{k}-u_{k}\right\|=\left(1-\beta_{k}\right)\left\|T u_{k}-u_{k}\right\| \leqslant\left\|T u_{k}-u_{k}\right\| \rightarrow 0,
$$

and hence

$$
\begin{aligned}
\left\|v_{k}-x_{k}\right\| & =\left\|\alpha_{k} \gamma V x_{k}+\gamma_{k} x_{k}+\left(\left(1-\gamma_{k}\right) I-\alpha_{k} \mu F\right) \tilde{u}_{k}-x_{k}\right\| \\
& =\left\|\alpha_{k}\left(\gamma \vee x_{k}-\mu F \tilde{u}_{k}\right)+\left(1-\gamma_{k}\right)\left(\tilde{u}_{k}-x_{k}\right)\right\| \\
& \leqslant \alpha_{k}\left\|\gamma V x_{k}-\mu F \tilde{u}_{k}\right\|+\left(1-\gamma_{k}\right)\left\|\tilde{u}_{k}-x_{k}\right\| \\
& \leqslant \alpha_{k}\left\|\gamma V x_{k}-\mu F \tilde{u}_{k}\right\|+\left(1-\gamma_{k}\right)\left(\left\|\tilde{u}_{k}-u_{k}\right\|+\left\|u_{k}-x_{k}\right\|\right) \\
& \leqslant \alpha_{k}\left\|\gamma V x_{k}-\mu F \tilde{u}_{k}\right\|+\left\|T u_{k}-u_{k}\right\|+\left\|u_{k}-x_{k}\right\| \rightarrow 0 .
\end{aligned}
$$


That is,

$$
\lim _{k \rightarrow \infty}\left\|\tilde{u}_{k}-u_{k}\right\|=0 \quad \text { and } \quad \lim _{k \rightarrow \infty}\left\|v_{k}-x_{k}\right\|=0 .
$$

Taking into consideration that $\left\|v_{k}-z_{k}\right\| \leqslant\left\|v_{k}-y_{k}\right\|+\left\|y_{k}-z_{k}\right\|$ and $\left\|z_{k}-x_{k}\right\| \leqslant\left\|z_{k}-v_{k}\right\|+\left\|v_{k}-x_{k}\right\|$, we deduce from (3.21) and (3.24) that

$$
\lim _{k \rightarrow \infty}\left\|v_{k}-z_{k}\right\|=0 \text { and } \lim _{k \rightarrow \infty}\left\|z_{k}-x_{k}\right\|=0 .
$$

It is clear from (3.21) and (3.24) that

$$
\lim _{k \rightarrow \infty}\left\|x_{k}-y_{k}\right\|=0 \text { and } \quad \lim _{k \rightarrow \infty}\left\|y_{k}-z_{k}\right\|=0 .
$$

Again by Proposition 2.1 (iii) and Lemma 3.3 we have

$$
\begin{aligned}
& \left\|P_{\mathrm{VI}(\Omega, \mathrm{B})}\left(\mathrm{y}_{\mathrm{k}}-\lambda A y_{\mathrm{k}}\right)-x_{\mathrm{k}+1}\right\| \\
& \leqslant\left\|\mathrm{P}_{\mathrm{VI}(\Omega, \mathrm{B})}\left(\mathrm{y}_{\mathrm{k}}-\lambda A y_{\mathrm{k}}\right)-\mathrm{P}_{\mathrm{VI}(\Omega, \mathrm{B})}\left(z_{\mathrm{k}}-\lambda A z_{\mathrm{k}}\right)\right\|+\left\|\mathrm{P}_{\mathrm{VI}(\Omega, \mathrm{B})}\left(z_{\mathrm{k}}-\lambda A z_{\mathrm{k}}\right)-x_{\mathrm{k}+1}\right\| \\
& \leqslant\left(1+\lambda \mathrm{L}_{1}\right)\left\|y_{k}-z_{k}\right\|+\alpha_{k}\left\|P_{\mathrm{VI}(\Omega, B)}\left(z_{k}-\lambda A z_{k}\right)-u\right\| \\
& +\beta_{\mathrm{k}}\left\|\mathrm{P}_{\mathrm{VI}(\Omega, \mathrm{B})}\left(z_{\mathrm{k}}-\lambda \mathrm{A} z_{\mathrm{k}}\right)-\mathrm{x}_{\mathrm{k}}\right\|+\bar{\epsilon}_{\mathrm{k}} \\
& \leqslant\left(1+\lambda \mathrm{L}_{1}\right)\left\|y_{\mathrm{k}}-z_{\mathrm{k}}\right\|+\alpha_{\mathrm{k}}\left\|\mathrm{P}_{\mathrm{VI}(\Omega, \mathrm{B})}\left(z_{\mathrm{k}}-\lambda \mathrm{A} z_{\mathrm{k}}\right)-u\right\|+\bar{\epsilon}_{\mathrm{k}} \\
& +\beta_{\mathrm{k}}\left\|\mathrm{P}_{\mathrm{VI}(\Omega, \mathrm{B})}\left(z_{\mathrm{k}}-\lambda A z_{\mathrm{k}}\right)-\mathrm{P}_{\mathrm{VI}(\Omega, \mathrm{B})}\left(\mathrm{y}_{\mathrm{k}}-\lambda A y_{\mathrm{k}}\right)\right\| \\
& +\beta_{k}\left\|P_{V I(\Omega, B)}\left(y_{k}-\lambda A y_{k}\right)-y_{k}\right\|+\beta_{k}\left\|y_{k}-x_{k}\right\| \\
& \leqslant\left(1+\lambda \mathrm{L}_{1}\right)\left\|y_{\mathrm{k}}-z_{\mathrm{k}}\right\|+\alpha_{\mathrm{k}}\left\|\mathrm{P}_{\mathrm{VI}(\Omega, \mathrm{B})}\left(z_{\mathrm{k}}-\lambda \mathrm{A} z_{\mathrm{k}}\right)-u\right\|+\bar{\epsilon}_{\mathrm{k}} \\
& +\beta_{k}\left(1+\lambda L_{1}\right)\left\|z_{k}-y_{k}\right\|+\beta_{k}\left\|P_{V I}(\Omega, B)\left(y_{k}-\lambda A y_{k}\right)-y_{k}\right\|+\beta_{k}\left\|y_{k}-x_{k}\right\| \text {. }
\end{aligned}
$$

Consequently, from (3.27), we have

$$
\begin{aligned}
& \left\|P_{V I}(\Omega, B)\left(y_{k}-\lambda A y_{k}\right)-y_{k}\right\| \leqslant\left\|P_{V I}(\Omega, B)\left(y_{k}-\lambda A y_{k}\right)-x_{k+1}\right\|+\left\|x_{k+1}-x_{k}\right\|+\left\|x_{k}-y_{k}\right\| \\
& \leqslant\left(1+\lambda \mathrm{L}_{1}\right)\left\|y_{\mathrm{k}}-z_{\mathrm{k}}\right\|+\alpha_{\mathrm{k}}\left\|\mathrm{P}_{\mathrm{VI}(\Omega, \mathrm{B})}\left(z_{\mathrm{k}}-\lambda A z_{\mathrm{k}}\right)-u\right\|+\bar{\epsilon}_{\mathrm{k}} \\
& +\beta_{k}\left(1+\lambda L_{1}\right)\left\|z_{k}-y_{k}\right\|+\beta_{k}\left\|P_{V I}(\Omega, B)\left(y_{k}-\lambda A y_{k}\right)-y_{k}\right\|+\beta_{k}\left\|y_{k}-x_{k}\right\| \\
& +\left\|x_{k+1}-x_{k}\right\|+\left\|x_{k}-y_{k}\right\| \\
& =\left(1+\beta_{k}\right)\left(1+\lambda L_{1}\right)\left\|y_{k}-z_{k}\right\|+\alpha_{k}\left\|P_{V I}(\Omega, B)\left(z_{k}-\lambda A z_{k}\right)-u\right\|+\bar{\epsilon}_{k} \\
& +\beta_{k}\left\|P_{V I}(\Omega, B)\left(y_{k}-\lambda A y_{k}\right)-y_{k}\right\|+\left(1+\beta_{k}\right)\left\|y_{k}-x_{k}\right\|+\left\|x_{k+1}-x_{k}\right\|,
\end{aligned}
$$

which immediately yields

$$
\begin{aligned}
\left\|P_{\mathrm{VI}(\Omega, \mathrm{B})}\left(y_{\mathrm{k}}-\lambda A y_{k}\right)-y_{k}\right\| \leqslant & \frac{1+\beta_{\mathrm{k}}}{1-\beta_{\mathrm{k}}}\left(1+\lambda \mathrm{L}_{1}\right)\left\|y_{\mathrm{k}}-z_{\mathrm{k}}\right\|+\frac{\alpha_{\mathrm{k}}}{1-\beta_{\mathrm{k}}}\left\|\mathrm{P}_{\mathrm{VI}(\Omega, \mathrm{B})}\left(z_{\mathrm{k}}-\lambda A z_{\mathrm{k}}\right)-u\right\|+\frac{\bar{\epsilon}_{\mathrm{k}}}{1-\beta_{\mathrm{k}}} \\
& +\frac{1+\beta_{\mathrm{k}}}{1-\beta_{\mathrm{k}}}\left\|y_{\mathrm{k}}-x_{\mathrm{k}}\right\|+\frac{1}{1-\beta_{\mathrm{k}}}\left\|\mathrm{x}_{\mathrm{k}+1}-x_{\mathrm{k}}\right\| .
\end{aligned}
$$

Since $\alpha_{k}+\beta_{k}+\gamma_{k}=1, \alpha_{k} \rightarrow 0, \beta_{k} \rightarrow \xi \in\left(\zeta, \frac{1}{2}\right], \bar{\epsilon}_{k} \rightarrow 0,\left\|y_{k}-z_{k}\right\| \rightarrow 0,\left\|y_{k}-x_{k}\right\| \rightarrow 0$, and $\left\|x_{k+1}-x_{k}\right\| \rightarrow 0$ (due to (3.17) and (3.26)), we conclude that

$$
\lim _{k \rightarrow \infty}\left\|P_{V I}(\Omega, B)\left(y_{k}-\lambda A y_{k}\right)-y_{k}\right\|=0 .
$$

From Proposition 2.1 (iii), it follows that

$$
\begin{aligned}
& \left\|\mathrm{P}_{\mathrm{VI}(\Omega, \mathrm{B})}\left(z_{\mathrm{k}}-\lambda A z_{\mathrm{k}}\right)-z_{\mathrm{k}}\right\| \leqslant\left\|\mathrm{P}_{\mathrm{VI}(\Omega, \mathrm{B})}\left(z_{\mathrm{k}}-\lambda A z_{\mathrm{k}}\right)-\mathrm{P}_{\mathrm{VI}(\Omega, \mathrm{B})}\left(\mathrm{y}_{\mathrm{k}}-\lambda A y_{\mathrm{k}}\right)\right\| \\
& +\left\|P_{\mathrm{VI}(\Omega, B)}\left(y_{k}-\lambda A y_{k}\right)-y_{k}\right\|+\left\|y_{k}-z_{k}\right\| \\
& \left.\leqslant\left(1+\lambda L_{1}\right)\left\|z_{k}-y_{k}\right\|+\| P_{V I(\Omega, B}\right)\left(y_{k}-\lambda A y_{k}\right)-y_{k}\|+\| y_{k}-z_{k} \| \\
& \leqslant\left\|P_{V I}(\Omega, B)\left(y_{k}-\lambda A y_{k}\right)-y_{k}\right\|+\left(2+\lambda L_{1}\right)\left\|y_{k}-z_{k}\right\| \text {. }
\end{aligned}
$$


Utilizing the last inequality we obtain from (3.26) and (3.28) that

$$
\lim _{\mathrm{k} \rightarrow \infty}\left\|\mathrm{P}_{\mathrm{VI}(\Omega, \mathrm{B})}\left(z_{\mathrm{k}}-\lambda \mathrm{A} z_{\mathrm{k}}\right)-z_{\mathrm{k}}\right\|=0 .
$$

Theorem 3.9. Suppose that the conditions (A1)-(A4) and (H1)-(H4) hold and that the conditions (B1) or (B2) hold. Then the two sequences $\left\{x_{k}\right\}$ and $\left\{z_{k}\right\}$ in Algorithm 3.2 converge strongly to the same point $x^{*} \in \operatorname{VI}(\operatorname{VI}(\Omega, B), A)$ provided $\left\|x_{k+1}-x_{k}\right\|=\mathrm{o}\left(\alpha_{k}\right)$, which is a unique solution to the VIP

$$
\left\langle(\mathrm{I}+\bar{\xi} \mu \mathrm{F}-\bar{\xi} \gamma \mathrm{V}) x^{*}-u, p-\chi^{*}\right\rangle \geqslant 0, \quad \forall p \in \operatorname{VI}(\mathrm{VI}(\Omega, \mathrm{B}), \mathrm{A}),
$$

where $\bar{\xi}=1-\xi \in\left[\frac{1}{2}, 1\right)$.

Proof. Note that Lemma 3.5 shows the boundedness of $\left\{x_{k}\right\}$. Since $H$ is reflexive, there is at least a weak convergence subsequence of $\left\{x_{k}\right\}$. First, let us assert that $\omega_{w}\left(x_{k}\right) \subset \operatorname{VI}(\operatorname{VI}(\Omega, B)$, A). As a matter of fact, take an arbitrary $w \in \operatorname{VI}(\operatorname{VI}(\Omega, \mathrm{B}), \mathrm{A})$. Then there exists a subsequence $\left\{x_{k_{i}}\right\}$ of $\left\{x_{k}\right\}$ such that $x_{k_{i}} \rightarrow w$. From (3.26), we know that $y_{k_{i}} \rightarrow w$. It is easy to see that the mapping $P_{V I(\Omega, B)}(I-\lambda A)$ : $\mathrm{C} \rightarrow \mathrm{VI}(\Omega, \mathrm{B}) \subset \mathrm{C}$ is nonexpansive because $\mathrm{P}_{\mathrm{VI}(\Omega, \mathrm{B})}$ is nonexpansive and $\mathrm{I}-\lambda \mathrm{A}$ is nonexpansive for $\beta$-inverse-strongly monotone mapping $A$ with $0<\lambda \leqslant 2 \beta$. So, utilizing Lemma 2.6 and (3.28), we obtain $w=\operatorname{PII}_{\mathrm{VI}(\Omega, \mathrm{B})}(w-\lambda A w)$, which leads to $w \in \operatorname{VI}(\operatorname{VI}(\Omega, \mathrm{B}), \mathrm{A})$. Thus, the assertion is valid.

It is clear that

$$
\langle(\mu F-\gamma V) x-(\mu F-\gamma V) y, x-y\rangle \geqslant(\mu \eta-\gamma l)\|x-y\|^{2}, \quad \forall x, y \in H .
$$

Hence, it follows from $0 \leqslant \gamma l<\tau \leqslant \mu \eta$ that $\mu \mathrm{F}-\gamma \mathrm{V}$ is $(\mu \eta-\gamma l)$-strongly monotone. In the meantime, it is clear that $\mu \mathrm{F}-\gamma \mathrm{V}$ is Lipschitzian with constant $\mu \mathrm{K}+\gamma \mathrm{l}>0$. We define the mapping $\Gamma: \mathrm{H} \rightarrow \mathrm{H}$ as below

$$
\Gamma x=(\mu \mathrm{F}-\gamma \mathrm{V}) x+\frac{1}{\bar{\xi}}(x-u), \quad \forall x \in \mathrm{H},
$$

where $u \in H$ and $\xi \in\left(\zeta, \frac{1}{2}\right]$. Then it is easy to see that $\Gamma$ is $\left(\mu \eta-\gamma l+\frac{1}{\xi}\right)$-strongly monotone and Lipschitzian with constant $\mu \mathrm{K}+\gamma \mathrm{l}+\frac{1}{\bar{\xi}}>0$. Thus, there exists a unique solution $x^{*} \in \operatorname{VI}(\operatorname{VI}(\Omega, \mathrm{B}), \mathrm{A})$ to the VIP

$$
\left\langle(\mu \mathrm{F}-\gamma \mathrm{V}) x^{*}+\frac{1}{\bar{\xi}}\left(x^{*}-u\right), p-x^{*}\right\rangle \geqslant 0, \quad \forall p \in \operatorname{VI}(\mathrm{VI}(\Omega, B), A) .
$$

Next, let us show that $x_{k} \rightarrow x^{*}$. Indeed, take an arbitrary $p \in \operatorname{VI}(\operatorname{VI}(\Omega, B), A)$. In terms of Algorithm 3.2 and Lemma 2.4, we conclude from (3.1), (3.3), and the $\beta$-inverse-strong monotonicity of $A$ with $\lambda \leqslant 2 \beta$, that

$$
\begin{aligned}
\left\|x_{k+1}-p\right\|^{2}= & \left\|\alpha_{k} u+\beta_{k} x_{k}+\gamma_{k} h_{k}-p\right\|^{2} \\
\leqslant & \left\|\beta_{k}\left(x_{k}-p\right)+\gamma_{k}\left(h_{k}-p\right)\right\|^{2}+2 \alpha_{k}\left\langle u-p, x_{k+1}-p\right\rangle \\
\leqslant & \beta_{k}\left\|x_{k}-p\right\|^{2}+\gamma_{k}\left\|h_{k}-p\right\|^{2}+2 \alpha_{k}\left\langle u-p, x_{k+1}-p\right\rangle \\
\leqslant & \beta_{k}\left\|x_{k}-p\right\|^{2}+\gamma_{k}\left(\left\|P_{V I}(\Omega, B)\left(z_{k}-\lambda A z_{k}\right)-p\right\|+\bar{\epsilon}_{k}\right)^{2}+2 \alpha_{k}\left\langle u-p, x_{k+1}-p\right\rangle \\
= & \beta_{k}\left\|x_{k}-p\right\|^{2}+\gamma_{k}\left(\left\|P_{V I}(\Omega, B)\left(z_{k}-\lambda A z_{k}\right)-P_{V I}(\Omega, B)(p-\lambda A p)\right\|+\bar{\epsilon}_{k}\right)^{2} \\
& +2 \alpha_{k}\left\langle u-p, x_{k+1}-p\right\rangle \\
\leqslant & \beta_{k}\left\|x_{k}-p\right\|^{2}+\gamma_{k}\left(\left\|(I-\lambda A) z_{k}-(I-\lambda A) p\right\|+\bar{\epsilon}_{k}\right)^{2}+2 \alpha_{k}\left\langle u-p, x_{k+1}-p\right\rangle \\
\leqslant & \beta_{k}\left\|x_{k}-p\right\|^{2}+\gamma_{k}\left(\left\|z_{k}-p\right\|+\bar{\epsilon}_{k}\right)^{2}+2 \alpha_{k}\left\langle u-p, x_{k+1}-p\right\rangle \\
= & \beta_{k}\left\|x_{k}-p\right\|^{2}+\gamma_{k}\left\|z_{k}-p\right\|^{2}+\gamma_{k} \bar{\epsilon}_{k}\left(2\left\|z_{k}-p\right\|+\bar{\epsilon}_{k}\right)+2 \alpha_{k}\left\langle u-p, x_{k+1}-p\right\rangle \\
\leqslant & \beta_{k}\left\|x_{k}-p\right\|^{2}+\gamma_{k}\left\|v_{k}-p\right\|^{2}+\gamma_{k} \bar{\epsilon}_{k}\left(2\left\|z_{k}-p\right\|+\bar{\epsilon}_{k}\right)+2 \alpha_{k}\left\langle u-p, x_{k+1}-p\right\rangle .
\end{aligned}
$$


Combining (3.20) and (3.30), we get

$$
\begin{aligned}
\left\|x_{k+1}-p\right\|^{2} \leqslant & \beta_{k}\left\|x_{k}-p\right\|^{2}+\gamma_{k}\left\|v_{k}-p\right\|^{2}+\gamma_{k} \bar{\epsilon}_{k}\left(2\left\|z_{k}-p\right\|+\bar{\epsilon}_{k}\right)+2 \alpha_{k}\left\langle u-p, x_{k+1}-p\right\rangle \\
\leqslant & \beta_{k}\left\|x_{k}-p\right\|^{2}+\gamma_{k}\left\{\left(1-\alpha_{k} \frac{\tau^{2}-(\gamma l)^{2}}{\tau}\right)\left\|x_{k}-p\right\|^{2}\right. \\
& -\left(1-\gamma_{k}-\alpha_{k} \tau\right)\left[r_{k}\left(2 \alpha-r_{k}\right)\left\|\mathcal{A} x_{k}-\mathcal{A} p\right\|^{2}+\left(1-\beta_{k}\right)\left(\beta_{k}-\zeta\right)\left\|u_{k}-T u_{k}\right\|^{2}\right] \\
& \left.+2 \alpha_{k}\left\langle(\gamma V-\mu F) p, v_{k}-p\right\rangle\right\}+\gamma_{k} \bar{\epsilon}_{k}\left(2\left\|z_{k}-p\right\|+\bar{\epsilon}_{k}\right)+2 \alpha_{k}\left\langle u-p, x_{k+1}-p\right\rangle \\
\leqslant & \beta_{k}\left\|x_{k}-p\right\|^{2}+\gamma_{k}\left\{\left(1-\alpha_{k} \frac{\tau^{2}-(\gamma l)^{2}}{\tau}\right)\left\|x_{k}-p\right\|^{2}+2 \alpha_{k}\left\langle(\gamma V-\mu F) p, v_{k}-p\right\rangle\right\} \\
& +\gamma_{k} \bar{\epsilon}_{k}\left(2\left\|z_{k}-p\right\|+\bar{\epsilon}_{k}\right)+2 \alpha_{k}\left\langle u-p, x_{k+1}-p\right\rangle \\
= & \left(\beta_{k}+\gamma_{k}-\alpha_{k} \gamma_{k} \frac{\tau^{2}-(\gamma l)^{2}}{\tau}\right)\left\|x_{k}-p\right\|^{2}+2 \alpha_{k} \gamma_{k}\left\langle(\gamma V-\mu F) p, v_{k}-x_{k+1}\right\rangle \\
& +\gamma_{k} \bar{\epsilon}_{k}\left(2\left\|z_{k}-p\right\|+\bar{\epsilon}_{k}\right)+2 \alpha_{k} \gamma_{k}\left\langle(\gamma V-\mu F) p+\frac{1}{\gamma_{k}}(u-p), x_{k+1}-p\right\rangle \\
\leqslant & \left(1-\alpha_{k} \gamma_{k} \frac{\tau^{2}-(\gamma l)^{2}}{\tau}\right)\left\|x_{k}-p\right\|^{2}+2 \alpha_{k}\|(\gamma V-\mu F) p\|\left(\left\|v_{k}-x_{k}\right\|+\left\|x_{k}-x_{k+1}\right\|\right) \\
& +\gamma_{k} \bar{\epsilon}_{k}\left(2\left\|z_{k}-p\right\|+\bar{\epsilon}_{k}\right)+2 \alpha_{k} \gamma_{k}\left\langle(\gamma V-\mu F) p+\frac{1}{\gamma_{k}}(u-p), x_{k+1}-p\right\rangle \\
\leqslant & \left\|x_{k}-p\right\|^{2}+2 \alpha_{k}\|(\gamma V-\mu F) p\|\left(\left\|v_{k}-x_{k}\right\|+\left\|x_{k}-x_{k+1}\right\|\right) \\
& +\gamma_{k} \bar{\epsilon}_{k}\left(2\left\|z_{k}-p\right\|+\bar{\epsilon}_{k}\right)+2 \alpha_{k} \gamma_{k}\left\langle(\gamma V-\mu F) p+\frac{1}{\gamma_{k}}(u-p), x_{k+1}-p\right\rangle,
\end{aligned}
$$

which immediately yields

$$
\begin{aligned}
& \left\langle(\mu \mathrm{F}-\gamma \mathrm{V}) p+\frac{1}{\gamma_{k}}(p-u), x_{k+1}-p\right\rangle \\
& \leqslant \frac{1}{2 \alpha_{k} \gamma_{k}}\left(\left\|x_{k}-p\right\|^{2}-\left\|x_{k+1}-p\right\|^{2}\right)+\frac{1}{\gamma_{k}}\|(\gamma V-\mu F) p\|\left(\left\|v_{k}-x_{k}\right\|+\left\|x_{k}-x_{k+1}\right\|\right) \\
& \quad+\frac{\bar{\epsilon}_{k}}{2 \alpha_{k}}\left(2\left\|z_{k}-p\right\|+\bar{\epsilon}_{k}\right) \\
& \leqslant \\
& \frac{\left\|x_{k}-x_{k+1}\right\|}{2 \alpha_{k} \gamma_{k}}\left(\left\|x_{k}-p\right\|+\left\|x_{k+1}-p\right\|\right)+\frac{1}{\gamma_{k}}\|(\gamma V-\mu F) p\|\left(\left\|v_{k}-x_{k}\right\|+\left\|x_{k}-x_{k+1}\right\|\right) \\
& \quad+\frac{\bar{\epsilon}_{k}}{2 \alpha_{k}}\left(2\left\|z_{k}-p\right\|+\bar{\epsilon}_{k}\right) .
\end{aligned}
$$

Since for any $w \in \omega_{w}\left(x_{k}\right)$ there exists a subsequence $\left\{x_{k_{i}}\right\}$ of $\left\{x_{k}\right\}$ such that $x_{k_{i}} \rightarrow w$, we deduce from (3.24), (3.32), $\frac{1}{\gamma_{k}} \rightarrow \frac{1}{\bar{\xi}}$, and $\left\|x_{k+1}-x_{k}\right\|=o\left(\alpha_{k}\right)$ that

$$
\begin{aligned}
\left\langle(\mu \mathrm{F}-\gamma \mathrm{V}) p+\frac{1}{\bar{\xi}}(p-u), w-p\right\rangle= & \lim _{i \rightarrow \infty}\left\langle(\mu \mathrm{F}-\gamma \mathrm{V}) p+\frac{1}{\gamma_{k_{i}}}(p-u), x_{k_{i}}-p\right\rangle \\
& \leqslant \limsup _{k \rightarrow \infty}\left\langle(\mu F-\gamma \mathrm{V}) p+\frac{1}{\gamma_{k}}(p-u), x_{k}-p\right\rangle \\
= & \limsup _{k \rightarrow \infty}\left\langle(\mu F-\gamma \mathrm{V}) p+\frac{1}{\gamma_{k}}(p-u), x_{k+1}-p\right\rangle \\
\leqslant & \limsup _{k \rightarrow \infty} \frac{\left\|x_{k}-x_{k+1}\right\|}{2 \alpha_{k} \gamma_{k}}\left(\left\|x_{k}-p\right\|+\left\|x_{k+1}-p\right\|\right) \\
& +\limsup _{k \rightarrow \infty} \frac{1}{\gamma_{k}}\|(\gamma V-\mu F) p\|\left(\left\|v_{k}-x_{k}\right\|+\left\|x_{k}-x_{k+1}\right\|\right)
\end{aligned}
$$




$$
\begin{aligned}
& +\limsup _{k \rightarrow \infty} \frac{\bar{\epsilon}_{k}}{2 \alpha_{k}}\left(2\left\|z_{k}-p\right\|+\bar{\epsilon}_{k}\right) \\
= & 0 .
\end{aligned}
$$

So, it follows that

$$
\left\langle(\mu \mathrm{F}-\gamma \mathrm{V}) p+\frac{1}{\bar{\xi}}(p-u), p-w\right\rangle \geqslant 0, \quad \forall p \in \operatorname{VI}(\mathrm{VI}(\Omega, B), A) .
$$

Since $w \in \omega_{w}\left(x_{k}\right) \subset \operatorname{VI}(\operatorname{VI}(\Omega, B), A)$, by Minty's lemma, we have

$$
\left\langle(\mu \mathrm{F}-\gamma \mathrm{V}) w+\frac{1}{\bar{\xi}}(w-u), p-w\right\rangle \geqslant 0, \quad \forall p \in \operatorname{VI}(\operatorname{VI}(\Omega, B), A)
$$

that is, $w$ is a solution of VIP (3.29). Utilizing the uniqueness of solutions of VIP (3.29), we get $w=x^{*}$, which hence implies that $\omega_{w}\left(x_{k}\right)=\left\{x^{*}\right\}$. Therefore, it is known that $\left\{x_{k}\right\}$ converges weakly to the unique solution $x^{*} \in \operatorname{VI}(\operatorname{VI}(\Omega, B)$, A ) of $\operatorname{VIP}(3.29)$.

Finally, let us show that $\left\|x_{k}-x^{*}\right\| \rightarrow 0$ as $k \rightarrow \infty$. Indeed, utilizing (3.31) with $p=x^{*}$, we have

$$
\begin{aligned}
\left\|x_{k+1}-x^{*}\right\|^{2} \leqslant & \left(1-\alpha_{k} \gamma_{k} \frac{\tau^{2}-(\gamma l)^{2}}{\tau}\right)\left\|x_{k}-x^{*}\right\|^{2}+2 \alpha_{k}\left\|(\gamma \mathrm{V}-\mu \mathrm{F}) x^{*}\right\|\left(\left\|v_{k}-x_{k}\right\|+\left\|x_{k}-x_{k+1}\right\|\right) \\
& +\gamma_{k} \bar{\epsilon}_{k}\left(2\left\|z_{k}-x^{*}\right\|+\bar{\epsilon}_{k}\right)+2 \alpha_{k} \gamma_{k}\left\langle(\gamma \mathrm{V}-\mu \mathrm{F}) x^{*}+\frac{1}{\gamma_{k}}\left(u-x^{*}\right), x_{k+1}-x^{*}\right\rangle \\
= & \left(1-\alpha_{k} \gamma_{k} \frac{\tau^{2}-(\gamma l)^{2}}{\tau}\right)\left\|x_{k}-x^{*}\right\|^{2} \\
& +\alpha_{k} \gamma_{k} \frac{\tau^{2}-(\gamma l)^{2}}{\tau} \times \frac{\tau}{\tau^{2}-(\gamma l)^{2}}\left[\frac{2}{\gamma_{k}}\left\|(\gamma \mathrm{V}-\mu \mathrm{F}) x^{*}\right\|\left(\left\|v_{k}-x_{k}\right\|+\left\|x_{k}-x_{k+1}\right\|\right)\right. \\
& \left.+\frac{\bar{\epsilon}_{k}}{\alpha_{k}}\left(2\left\|z_{k}-x^{*}\right\|+\bar{\epsilon}_{k}\right)+2\left\langle(\gamma \mathrm{V}-\mu \mathrm{F}) x^{*}+\frac{1}{\gamma_{k}}\left(u-x^{*}\right), x_{k+1}-x^{*}\right\rangle\right] .
\end{aligned}
$$

Since $\alpha_{k} \rightarrow 0, \alpha_{k}+\beta_{k}+\gamma_{k}=1, \quad \sum_{k=0}^{\infty} \alpha_{k}=\infty, \beta_{k} \rightarrow \xi \in\left(\zeta, \frac{1}{2}\right], \bar{\epsilon}_{k}=o\left(\alpha_{k}\right)$, and $x_{k} \rightarrow x^{*}$, from (3.17) and (3.21) we conclude that $\sum_{k=0}^{\infty} \alpha_{k} \gamma_{k} \frac{\tau^{2}-(\gamma l)^{2}}{\tau}=\infty$ and

$$
\begin{aligned}
& \limsup _{k \rightarrow \infty} \frac{\tau}{\tau^{2}-(\gamma l)^{2}}\left[\frac{2}{\gamma_{k}}\left\|(\gamma \mathrm{V}-\mu \mathrm{F}) x^{*}\right\|\left(\left\|v_{k}-x_{k}\right\|+\left\|x_{k}-x_{k+1}\right\|\right)\right. \\
& \left.+\frac{\bar{\epsilon}_{k}}{\alpha_{k}}\left(2\left\|z_{k}-x^{*}\right\|+\bar{\epsilon}_{k}\right)+2\left\langle(\gamma V-\mu F) x^{*}+\frac{1}{\gamma_{k}}\left(u-x^{*}\right), x_{k+1}-x^{*}\right\rangle\right] \leqslant 0 .
\end{aligned}
$$

Therefore, applying Lemma 2.11 to (3.33), we obtain that $\left\|x_{k}-x^{*}\right\| \rightarrow 0$ as $k \rightarrow \infty$. Utilizing (3.25) we also obtain that $\left\|z_{k}-x^{*}\right\| \rightarrow 0$ as $k \rightarrow \infty$. This completes the proof.

\section{Acknowledgment}

This work was supported by the Innovation Program of Shanghai Municipal Education Commission (15ZZ068), Ph.D. Program Foundation of Ministry of Education of China (20123127110002) and Program for Shanghai Outstanding Academic Leaders in Shanghai City (15XD1503100). This study was supported by the grant MOST 105-2115-M-037-001, and the grand from Research Center for Nonlinear Analysis and Optimization, Kaohsiung Medical University, Kaohsiung, Taiwan.

\section{References}

[1] P. N. Anh, J. K. Kim, L. D. Muu, An extragradient algorithm for solving bilevel pseudomonotone variational inequalities, J. Global Optim., 52 (2012), 627-639. 1, 1.2, 1 
[2] L.-C. Ceng, S. Al-Homidan, Q. H. Ansari, J.-C. Yao, An iterative scheme for equilibrium problems and fixed point problems of strict pseudo-contraction mappings, J. Comput. Appl. Math., 223 (2009), 967-974. 1, 1, 1

[3] L.-C. Ceng, Q. H. Ansari, J.-C. Yao, Iterative methods for triple hierarchical variational inequalities in Hilbert spaces, J. Optim. Theory Appl., 151 (2011), 489-512. 1

[4] L.-C. Ceng, Q. H. Ansari, J.-C. Yao, An extragradient method for solving split feasibility and fixed point problems, Comput. Math. Appl., 64 (2012), 633-642. 1

[5] L.-C. Ceng, Q. H. Ansari, J.-C. Yao, Relaxed extragradient methods for finding minimum-norm solutions of the split feasibility problem, Nonlinear Anal., 75 (2012), 2116-2125. 1

[6] L.-C. Ceng, Q. H. Ansari, J.-C. Yao, Relaxed hybrid steepest-descent methods with variable parameters for triplehierarchical variational inequalities, Appl. Anal., 91 (2012), 1793-1810. 1

[7] L.-C. Ceng, S.-M. Guu, J.-C. Yao, Hybrid iterative method for finding common solutions of generalized mixed equilibrium and fixed point problems, Fixed Point Theory Appl., 2012 (2012), 19 pages. 1

[8] L.-C. Ceng, J.-C. Yao, A hybrid iterative scheme for mixed equilibrium problems and fixed point problems, J. Comput. Appl. Math., 214 (2008), 186-201. 1, 2.3

[9] F. Facchinei, J.-S. Pang, Finite-dimensional variational inequalities and complementarity problems, Vol. I, II, Springer Series in Operations Research, Springer-Verlag, New York, (2003). 1

[10] R. Glowinski, Numerical methods for nonlinear variational problems, Springer Series in Computational Physics, Springer-Verlag, New York, (1984). 1

[11] K. Goebel, W. A. Kirk, Topics in metric fixed point theory, Cambridge Studies in Advanced Mathematics, Cambridge University Press, Cambridge, (1990). 2.6, 2.12

[12] H. Iiduka, Iterative algorithm for solving triple-hierarchical constrained optimization problem, J. Optim. Theory Appl., 148 (2011), 580-592. 1, 1.5, 1.6, 1

[13] I. V. Konnov, Regularization method for nonmonotone equilibrium problems, J. Nonlinear Convex Anal., 10 (2009), 93-101. 1

[14] G. M. Korpelevich, The extragradient method for finding saddle points and other problems, Matecon, 12 (1976), 747-756. 1

[15] J.-L. Lions, Quelques méthodes de résolution des problémes aux limites non linéaires, (French) Dunod; Gauthier-Villars, Paris, (1969). 1

[16] G. Marino, H.-K. Xu, Weak and strong convergence theorems for strict pseudo-contractions in Hilbert spaces, J. Math. Anal. Appl., 329 (2007), 336-346. 1, 2.7

[17] J. Outrata, M. Kočvara, J. Zowe, Nonsmooth approach to optimization problems with equilibrium constraints, Theory, applications and numerical results, Nonconvex Optimization and its Applications, Kluwer Academic Publishers, Dordrecht, (1998). 1

[18] J.-W. Peng, J.-C. Yao, A new hybrid-extragradient method for generalized mixed equilibrium problems, fixed point problems and variational inequality problems, Taiwanese J. Math., 12 (2008), 1401-1432. 1, 1, 1

[19] M. Solodov, An explicit descent method for bilevel convex optimization, J. Convex Anal., 14 (2007), 227- 237. 1

[20] T. Suzuki, Strong convergence of Krasnoselskii and Mann's type sequences for one-parameter nonexpansive semigroups without Bochner integrals, J. Math. Anal. Appl., 305 (2005), 227-239. 3.6

[21] S. Takahashi, W. Takahashi, Viscosity approximation methods for equilibrium problems and fixed point problems in Hilbert spaces, J. Math. Anal. Appl., 331 (2007), 506-515. 1, 1, 1

[22] S. Takahashi, W. Takahashi, Strong convergence theorem for a generalized equilibrium problem and a nonexpansive mapping in a Hilbert space, Nonlinear Anal., 69 (2008), 1025-1033. 1

[23] H.-K. Xu, T. H. Kim, Convergence of hybrid steepest-descent methods for variational inequalities, J. Optim. Theory. Appl., 119 (2003), 185-201. 2.10, 2.11

[24] M. H. Xu, M. Li, C. C. Yang, Neural networks for a class of bi-level variational inequalities, J. Global Optim., 44 (2009), 535-552. 1

[25] I. Yamada, The hybrid steepest descent method for the variational inequality problem over the intersection of fixed point sets of nonexpansive mappings, Inherently parallel algorithms in feasibility and optimization and their applications, Haifa, (2000), 473-504, Stud. Comput. Math., North-Holland, Amsterdam, 8 (2001). 1

[26] Y.-H. Yao, Y.-C. Liou, S. M. Kang, Approach to common elements of variational inequality problems and fixed point problems via a relaxed extragradient method, Comput. Math. Appl., 59 (2010), 3472-3480. 1, 2.8

[27] Y.-H. Yao, Y.-C. Liou, J.-C. Yao, An extragradient method for fixed point problems and variational inequality problems, J. Inequal. Appl., 2007 (2007), 12 pages. 1, 3

[28] H. Zegeye, N. Shahzad, Y.-H. Yao, Minimum-norm solution of variational inequality and fixed point problem in Banach spaces, Optimization, 64 (2015), 453-471. 1

[29] L.-C. Zeng, M. M. Wong, J.-C. Yao, Strong convergence of relaxed hybrid steepest-descent methods for triple hierarchical constrained optimization, Fixed Point Theory Appl., 2012 (2012), 24 pages. 1

[30] L.-C. Zeng, J.-C. Yao, Modified combined relaxation method for general monotone equilibrium problems in Hilbert spaces, J. Optim. Theory Appl., 131 (2006), 469-483. 1

[31] L.-C. Zeng, J.-C. Yao, Strong convergence theorem by an extragradient method for fixed point problems and variational inequality problems, Taiwanese J. Math., 10 (2006), 1293-1303. 1 